\title{
Nanotheranostics - a review of recent publications
}

This article was published in the following Dove Press journal:

International Journal of Nanomedicine

24 August 2012

Number of times this article has been viewed

\section{Li-Sheng Wang Min-Chieh Chuang Ja-an Annie Ho \\ Department of Biochemical Science and Technology, National Taiwan University, Taipei, Taiwan}

\begin{abstract}
Theranostics is referred to as a treatment strategy that combines therapeutics with diagnostics, aiming to monitor the response to treatment and increase drug efficacy and safety, which would be a key part of personalized medicine and require considerable advances in predictive medicine. Theranostics associates with both a diagnosis that tests patients for possible reactions to taking new medication and targeted drug delivery based on the test results. Emerging nanotechnology provides a great deal of opportunity to design and develop such combination agents, permitting the delivery of therapeutics and concurrently allowing the detection modality to be used not only before or after but also throughout the entire treatment regimen. The introduction of nanotheranostics into routine health care has still a long way to go, since evaluations on cytotoxicity, genotoxicity, and immunotoxicity of prospective nanotheranostics, demonstration of cost-effectiveness, and availability of appropriate accessible testing systems are still required. An extensive review, from a chemistry point of view, of the recent development of nanotheranostics and its in vitro and in vivo applications are herein presented.
\end{abstract}

Keywords: theranostics, therapeutics, diagnostics, nanomaterial

\section{Introduction}

The term "theranostics" was coined to define a proposed methodology that combined the modalities of the therapeutic and diagnostic. ${ }^{1}$ Its goal is to develop specific and individualized therapeutic strategies towards personalized medicine, in light of the fact that acceptable efficacy of specific treatment could be achieved for only very few patients. By combining therapeutic and diagnostic capability into one single agent, a new protocol is anticipated to tailor a treatment based on the test results, thereby providing more specific and efficient systems for the curing of disease. Such combination agents are materials capable of detecting and treating diseases in one single procedure. Emerging nanotechnology is offering great opportunities to design and generate such agents, wherein the detection modality is extensively allowed to run not only before or after but also through the treatment regimen.

A thoroughly successful realization of such nanotheranostic agents relies on several inherent properties of nanoparticles (NPs). One of the most promising characteristics of utilizing NPs as theranostic agents is the possibility to localize them in specific sites of diseases and mitigate undesired side effects. Due to the nanometric size of these nanomaterials, the chance that they are cleared through the kidneys is significantly reduced, thereby extending circulation time in blood. Furthermore, tumor blood vessels tend to be irregularly dilated and leaky, and due to this feature nanosized particles can easily extravasate from the blood pool into tumor tissues and be retained as a result 
of poor lymphatic drainage. This selective accumulation of nanosized particles in tumor tissues is termed the enhanced permeability and retention effect.

An additional advantageous property of NPs also involves the high surface-area-to-volume ratio, thereby yielding a high loading capacity of therapeutic drugs and imaging agents. Moreover, the greater surface area can be functionalized with stabilizing agents (eg, polyethylene glycol [PEG]) and ligands aimed at cloaking and targeting purposes. Many nanomaterials are already imaging agents (eg, magnetic NPs for magnetic resonance imaging [MRI]), thereby readily being upgraded to theranostic agents by incorporating the therapeutic utility within. Since multiple types of molecules can be incorporated onto a single nanoparticle surface concurrently, the same nanoscale complex can achieve multiple functions.

With regard to the aim of reducing systemic toxicity, selectively targeting diseased tissues has been one of the main challenges in drug delivery. A qualified targeting approach facilitates accumulation of theranostic agents at the targeted region of interest, thereby raising the local concentration of therapeutic agent, and additionally increases the target-tobackground contrast in the imaging. Meanwhile, precluding theranostic agents from clearance by innate immunosystem recognition to secure sufficiently long circulation is essential for reaching targets efficiently.

This review gives a summary of selected recent development (2009-2012) on nanotheranostics. We present various nanotheranostic agents, categorized according to their core materials, along with significant advances on both therapeutic and diagnostic (imaging) aspects. Table 1 summarizes common approaches in both therapeutics and imaging presented in matrix form on the basis of the utilized technologies described by the selected methods. The bracketed numbers indicate studies in the reference section. As can be interpreted from the matrix of analyzed results, optical and MRI are the preferable modalities performed for imaging functionality, through use of near-infrared light (NIR) emission and magnetic agents, respectively. These two imaging strategies are mostly integrated with either chemo- or photothermal therapy (PTT) to produce multiple functions that have been demonstrated by employing diverse core nanomaterials. Gold and magnetic NPs have been used extensively in PTT and MRI, due to the properties of these nanomaterials. However, focusing on imaging alone without therapy functionality will not be the main point of this article, and such discussions are available in several excellent reviews. ${ }^{2,3}$ Instead, the design and development of theranostic agents, particularly from a chemistry point of view, and possible applications will be discussed and addressed herein.

\section{Gold-based nanomaterials}

Due to their superior biocompatibility and well-established strategies for surface modification (ie, gold-thiol bonding), gold-based nanomaterials have been investigated as multifunctional probes. The unique optical and photothermal characteristics of gold nanomaterials enable them not only to be applied as sensing utilities but also to induce photothermal effects for therapeutic purposes. The localized surface plasmon resonance (LSPR) of gold nanomaterials can be adjusted by tuning their morphology; gold $(\mathrm{Au}) \mathrm{NP}$, nanorod (AuNR), nanoshell, and nanocage exhibit distinctive optical and thermal properties, which can readily upgrade gold nanomaterials to be prospective theranostic agents.

Gold nanomaterial provides a versatile platform for simultaneously carrying therapeutics and diagnostics on its surface via gold-thiol bonding. Subsequently, those attached drugs can be intracellularly released from gold nanocarriers by exchange reactions with cytosolic glutathione (abundant glutathione presented in cell, $\sim 10 \mathrm{mM}) .{ }^{4}$ In a recent report described by Heo et $\mathrm{al}^{5}$ AuNP modified with multiple ligands, such as PEG, biotin, paclitaxel (PTX), and rhodamine $\mathrm{B}$-linked $\beta$-cyclodextrin on the surface was demonstrated to be a useful theranostic agent for cancer therapy without a cytotoxic effect on normal cells.

Lacking intrinsic fluorescent attributes, regular-sized AuNP was rarely used as an optical imaging agent (excluding ultrasmall $\mathrm{Au}$ clusters). However, due to its higher atomic number and X-ray absorption coefficient than iodine, the feasibility of AuNP as contrast agent in computed tomography (CT) imaging was studied. ${ }^{6}$ In addition, Kim et al presented novel multifunctional AuNP for targeted molecular CT imaging and therapy of prostate cancer, ${ }^{7}$ in which a prostate-specific membrane antigen (PSMA) RNA aptamer that bound to PSMA was functionalized onto the surface of AuNP. It was confirmed in the study that the as-fabricated PSMA aptamer-conjugated AuNP demonstrated greater CT intensity toward targeted LNCaP cells than that of nontargeted PC3 cells. Moreover, PSMA aptamer-conjugated AuNP loaded with the anticancer drug doxorubicin (DOX) was notably more effective against targeted LNCaP than against nontargeted PC3 cells.

Additionally, the high X-ray absorption coefficient makes gold nanomaterial not only a good CT imaging contrast agent but also a great radiotherapy sensitizer. As we already know, 
Table I Summary of therapeutic methods, diagnostic strategies, and corresponding nanomaterials discussed in this review

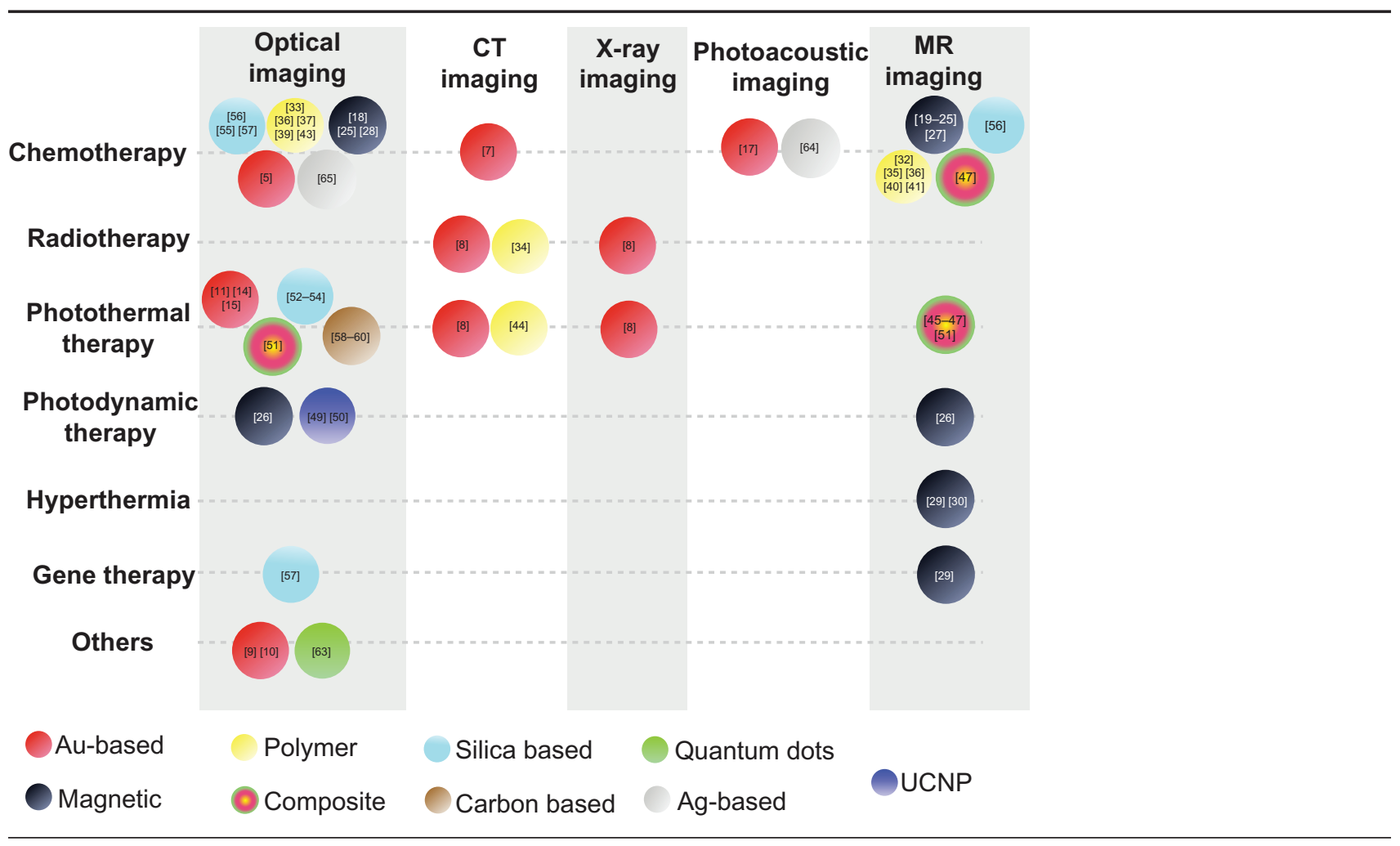

Abbreviations: CT, computed tomography; MR, magnetic resonance; UCNP, up-conversion nanoparticle.

the greatest challenge for radiation therapy (RT) is to reach the highest probability of cure without significant morbidity. Targeted delivery of radiotherapy sensitizer to the tumor site seems to improve the accuracy of radiation treatment, so that more targeted doses can be delivered while reducing the impact on neighboring tissues. In a recent study, Huang et al synthesized a folic acid-conjugated silica-modified gold nanorod ( $\left.\mathrm{GNR}-\mathrm{SiO}_{2}-\mathrm{FA}\right)$ and demonstrated its efficacy on X-ray RT and PTT. ${ }^{8}$ The authors claimed that such multifunctional nanoprobes were designed to possess various functions in tumor targeting, imaging, and selective therapy, offering great potential for highly selective targeting and enhanced RT and PTT effects on MGC803 gastric cancer cells.

Instead of a direct utilization of AuNP as an imaging contrast or photothermal agent, Lukianova-Hleb et al developed a method based on the AuNP-generated transient photothermal vapor nanobubble, the so called plasmonic nanobubble (PNB). ${ }^{9}$ The intracellular PNBs were able to be optically generated and controlled via laser fluence $\left(\mathrm{J} / \mathrm{cm}^{2}\right)$ after delivery and clusterization of the AuNP to the target cells. A noninvasive amplification of the optical scattering amplitude with the PNB (relative to that of AuNP), selective mechanical and speedy damage to specific cells with bigger $\mathrm{PNB}$, and optical guidance of therapy via the damage-specific signals of the bubbles were presented. A study conducted by Wagner et $\mathrm{al}^{10}$ once again confirmed that PNB was capable of serving as an in vivo tunable theranostic cellular agent in zebrafish hosting prostate cancer xenografts.

To diagnose cancer, visualization of carcinoid tumors by targeted imaging probe is not the only way. As shown in Figure 1, Yi et al designed and developed a novel probe - matrix metalloprotease (MMP)-sensitive gold nanorod (MMP-AuNR) - for cancer diagnosis and therapy (Figure 1A). ${ }^{11}$ MMP is a family of zinc-dependent proteins, and the production of MMP is distinctly associated with cancer metabolism. ${ }^{12}$ Therefore, the imaging of MMP could provide valuable information about cancer diagnosis. By conjugating Cy5.5 with a known peptide that can be degraded by MMP, ${ }^{13}$ and subsequently putting modified Cy5.5-peptide complex onto the surface of AuNR, a fluorescent probe for detecting MMP was successfully fabricated, which could be used to determine the progress of cancer by monitoring the fluorescence recovery of quenched Cy5.5 (Figure 1B, D, and E). In addition, the photothermal effect induced by MMP-AuNR was able to raise the media temperature to $60^{\circ} \mathrm{C}$ (Figure $1 \mathrm{C}$ and $\mathrm{F}$ ), leading to the damage of tumor tissue (Figure 1G). In a recent study carried out by $\mathrm{Hu}$ et $\mathrm{a} \mathrm{l}^{14}$ the photothermal effect of AuNR was efficiently improved by a surrounding silver shell (AuNR-in-shell). The 
A
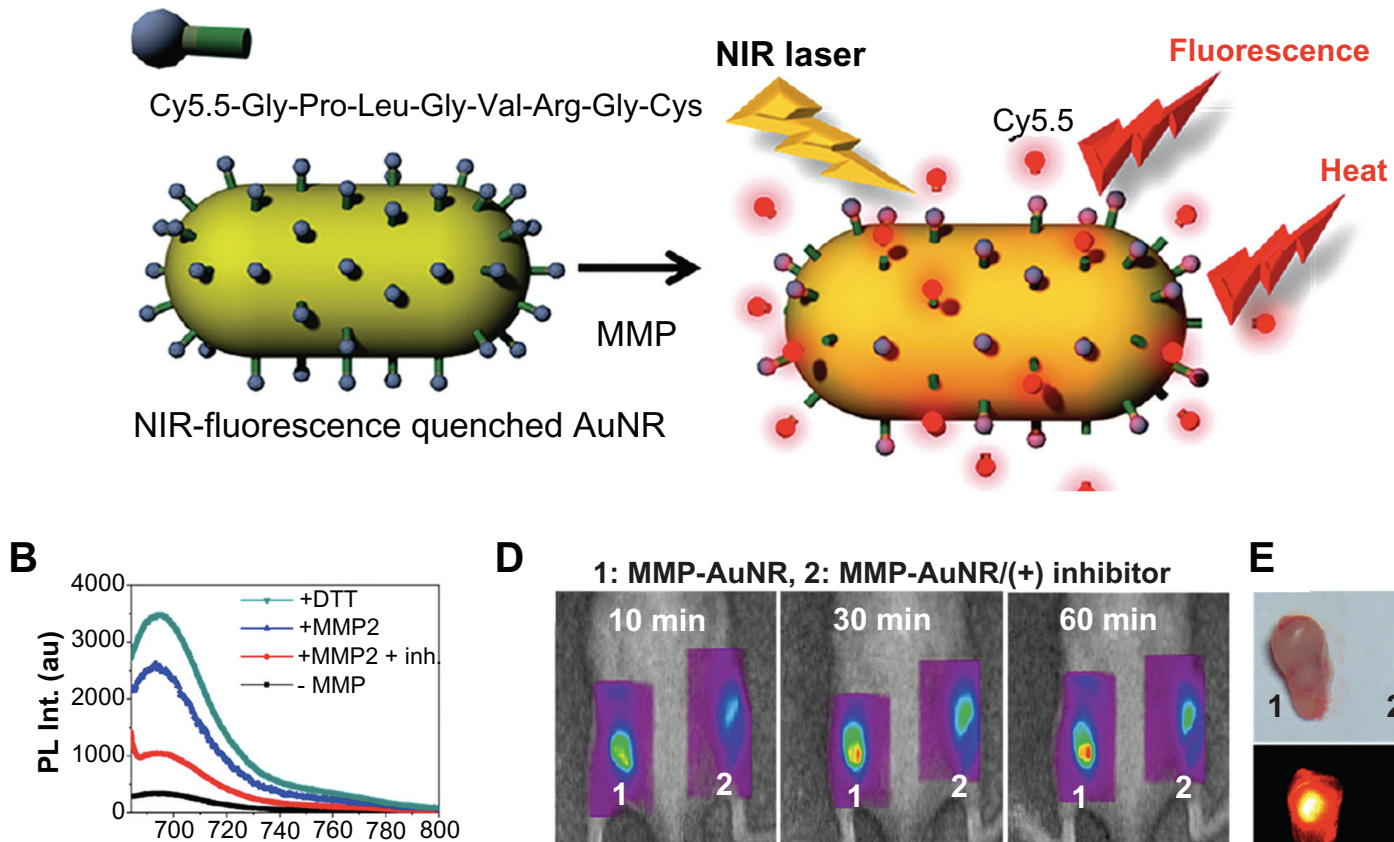

D
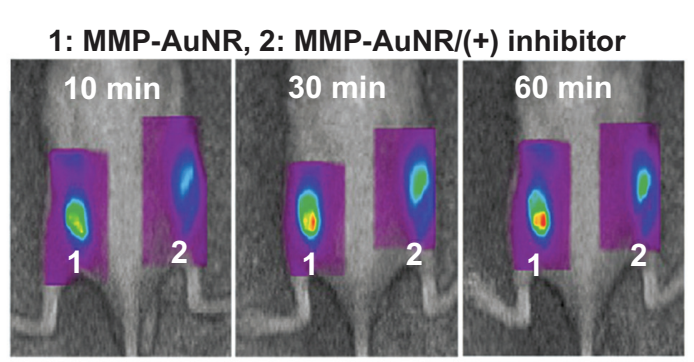

E
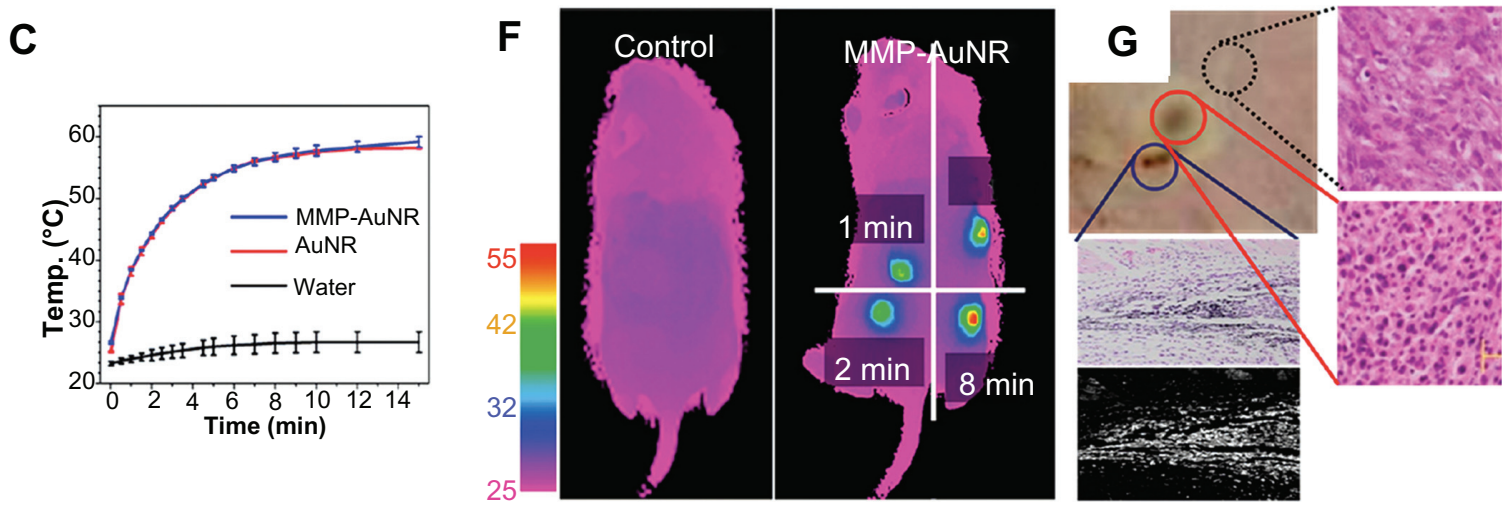

Figure I (A) Schematic representation of MMP-AuNR for simultaneous imaging and photothermal therapy; (B) fluorescence spectra of MMP-AuNR in the presence of MMP-2 enzyme and dithiothreitol; (C) temperature increase of MMP-AuNR as a function of laser irradiation time; (D) NIRF tomographic images of SCC-7 tumor-bearing mice after intratumoral injection of the MMP-AuNR probe without (I) and with (2) inhibitor; (E) optical and NIRF images of excised tumor after injection of MMP-AuNR without and with MMP-2 inhibitor; (F) infrared thermal images of tumor-bearing mice according to different laser irradiation times; (G) optical images of exterior of SCC-7 tumor irradiated by laser; histology (right) and dark-field image (lower) of tumor after treatment are also presented.

Reprinted with permission from Yi DK, Sun IC, Ryu JH, et al. Matrix metalloproteinase sensitive gold nanorod for simultaneous bioimaging and photothermal therapy of cancer. Bioconjug Chem. Copyright (C) 2010 American Chemical Society. "I

Abbreviations: MMP-AuNR, matrix metalloprotease-sensitive gold nanorod; SCC, squamous cell carcinoma; NIRF, near-infrared fluorescence.

authors found that AuNR-in-shell nanostructures destroyed malignant cells at half the laser power needed for AuNR, suggesting that in addition to AuNR, AuNR-in-shell was also a potential candidate in theranostic applications. In addition, $\mathrm{Au}$ nanocubes were also demonstrated for use in cell imaging and PTT. In 2009, Wu et al ${ }^{15}$ described unique optical properties of the high photoluminescence quantum yield and high extinction coefficients of Au nanocubes, allowing cell imaging of human liver cancer cells (QGY) and human embryo kidney cells (293T) with a common method of single-photon excitation and similar photo-killing efficiency as compared to AuNR.
In regard to the penetration depth of electromagnetic wave, spherical gold nanomaterials are not suitable to be used as an imaging or therapeutic probe in vivo (because their LSPR bands are in the visible-light region). In order to change the LSPR peak to NIR range, nonspherical or hollow formats of gold nanomaterials were thus designed. Xia's group developed a new Au nanocage that not only possessed an NIR photothermal effect similar to AuNR but also could be applied as a contrast agent for photoacoustic tomography owing to its high optical absorption in the NIR region. ${ }^{16}$ Moreover, Moon et al ${ }^{17}$ revealed a controlled 
releasable Au nanocage by filling the hollow interiors of gold nanocages with a phase-change material such as 1-tetradecanol, which has a melting point of $38^{\circ} \mathrm{C}-39^{\circ} \mathrm{C}$. When NIR laser or high-intensity focused ultrasound was applied to raise the temperature of the medium (higher than the melting temperature of the phase-change material), the encapsulated molecules were released from the inside of the Au nanocage. Au nanocage showed great controllability of release profile by direct exposure of heat or high-intensity focused ultrasound methods, suggesting a new format of gold nanomaterial-based theranostic agents.

\section{Magnetic nanomaterials}

While studies regarding the encapsulation/loading of magnetic NPs in a variety of polymers are discussed elsewhere in this article, the following magnetic theranostic nanocarrier scopes the various vesicles utilizing magnetic materials as the core.

Magnetic NP-based theranostics is advantageous due to the NPs' inherent biocompatibility and cost-effectiveness, along with their magnetic property as nanostructured contrast probes for MRI. Among the magnetic NPs, superparamagnetic iron oxide nanoparticles (IONPs), mainly magnetite and maghemite, are the most commonly used nanomaterials. In order to confer colloidal suspendability to the particles, hydrophilic polymers are added to passivate the nanocrystal surface that would typically protect particles from aggregation. A number of polymers, including dextran, dendrimer, polyaniline, and polyvinylpyrrolidone, have been utilized aimed at such purposes; in particular, dextran and its derivatives have been intensively studied. In addition, specific polymers coated on magnetic NPs were found to facilitate drug encapsulation and offer functional moiety for covalent conjugation.

Encapsulation of drug onto polymer-coated magnetic NPs has been realized in a variety of fashions. For instance, an earlier work simply utilized poly(acrylic acid) (PAA) to coencapsulate a lipophilic NIR dye and the anticancer drug taxol within hydrophobic pockets, resulting in a theranostic nanocarrier for dual fluorescence- and MR-based imaging and monitoring of drug delivery. Folate, furthermore, was conjugated onto PAA-IONPs, yielding targeting functionality aimed at targeted killing of folate receptor-overexpressing cancer cells, demonstrated by optical/MR imaging. ${ }^{18}$ In a later study by Rastogi et al, ${ }^{19}$ three polymers with varied functionalities surrounding the superparamagnetic IONPs to generate a thermosensitive nanocarrier were presented. While $N$-isopropylacrylamide (NIPAAM) acted as a temperature-sensitive polymer for hyperthermia capability, acrylic acid was utilized to enhance conjugation to an iron surface as well as tune critical temperature by its hydrophilic nature. In addition, PEG methacrylate provided a stealth coating, thereby increasing the circulation time and enabling reactive groups $(-\mathrm{OH})$ for folic acid coupling. Such polymeric nanocomposites of $\sim 200 \mathrm{~nm}$ in size showed darker transverse relaxation time (T2)-weighted images than the control, examined in phantom agar gels. Taking advantage of the thermoresponsive property of NIPAAM has resulted in precisely controlled release of DOX at hyperthermia temperatures (a total of $72 \% \pm 5.25 \%$ in 48 hours), nearly 2.5-fold higher than that for normal physiological condition.

Instead of using three polymers for distinct functionalities, both hydrophobic and hydrophilic characteristics are concurrently available in an amphiphilic polymer. Pluronic F127, nonionic triblockcopolymers composed of a central hydrophobic chain of polyoxypropylene (poly[propylene oxide]) flanked by two hydrophilic chains of polyoxyethylene (poly[ethylene oxide]), was coated, along with $\beta$-cyclodextrin, onto magnetic NPs, allowing an efficient encapsulation of the anti-cancer drug currcumin. ${ }^{20}$ An optimized formula named F127250 yielded beneficial characteristics, including smaller particle size, relatively lower protein binding, higher drug-loading efficacy, and superior uptake of particles in cancer cells without hampering inherent magnetization characteristics. Many folds of imaging-contrast properties and superior hyperthermia effect were furthermore provided over time under alternating magnetic field by the drugloaded formulation of F127250, compared to pure magnetic NPs and $\beta$-cyclodextrin-coated NPs. Similar work with a utilization of Pluronic F127 was also reported, ${ }^{21}$ in which polymer was coated on magnetic NPs to provide prolonged contrast property in MRI with higher loading of hydrophobic anticancer agents for sustained drug delivery. Specifically, five different NIR dyes were systematically investigated to determine, in mice, the long-term biodistribution and tumor localization with and without an external magnetic field. Such magnetic nanocarriers localized slowly in the tumor, reaching a peak at 48 hours post-injection before gradually declining over the next 11 days.

In addition to the competence of the thermoresponsive polymeric stated above, Lim et $\mathrm{al}^{22}$ revealed an intelligent nanoplatform of herceptin (antibody of HER2)-modified, $\mathrm{pH}$-sensitive drug delivering magnetic NPs aimed at effective cancer therapy guided by molecular imaging (as indicated in Figure 2). The authors employed $\alpha$-pyrenyl- $\omega$-carboxyl PEG to encapsulate magnetic resonance-sensitive $\mathrm{MnFe}_{2} \mathrm{O}_{4}$ 

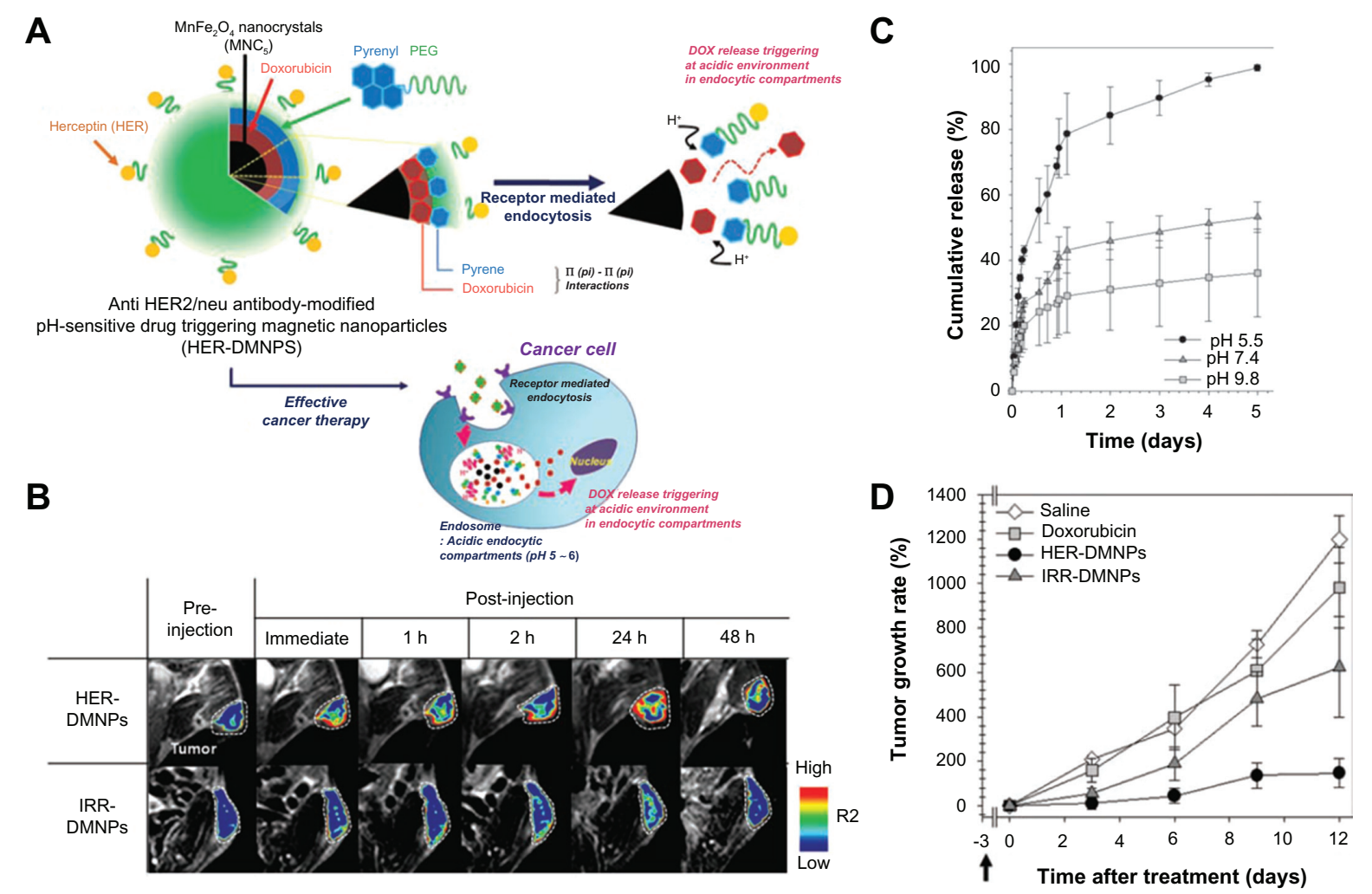

Figure 2 (A) Schematic illustration of anti-HER2/neu antibody-modified pH-sensitive drug-releasing magnetic nanoparticles (HER-DMNPs) for cancer therapy followed by magnetic resonance imaging (MRI); (B) color-coded T2-weighted MR images of tumor-bearing mice after the intravenous injection of HER-DMNPs and IRR-DMNPs at various time intervals, respectively; tumor regions are indicated with a white-dashed boundary; $(\mathbf{C}) \mathrm{pH}$-sensitive drug release profiles of the DMNPs over 5 days (black circle, $\mathrm{pH}$ 5.5; dark-gray triangle, $\mathrm{pH}$ 7.4; gray square, $\mathrm{pH}$ 9.8); (D) comparative therapeutic efficacy study in the in vivo model (black circle, HER-DMNPs; dark-gray triangle, IRR-DMNPs; gray square, doxorubicin; white diamond, saline); black arrow indicates the day of cancer cells (NIH3T6.7 cells) implantation in mice.

Reprinted from Adv Mater. 20I I;23(2I):2436-2442. Lim EK, Huh YM, Yang J, Lee K, Suh JS, Haam S. pH-Triggered drug-releasing magnetic nanoparticles for cancer therapy guided by molecular imaging by MRI. (C) 201 I with permission from Wiley. ${ }^{22}$

nanocrystals and DOX by the nanoemulsion method, triggering the release of DOX under proton-rich conditions (Figure 2A). DOX coupled with the pyrene groups via $\pi-\pi$ interaction was weakened under the intracellular (acidic) conditions of cancer cells (pH5) to cause a rapid release (Figure 2C). By MRI examination (Figure 2B), successful targeting of NIH3T6.7 cells, and a HER2-overexpressed mouse fibroblast cell line, was demonstrated by this theranostic nanocomposite, which subsequently suppressed cell growth by the synergistic therapeutic efficacy of DOX and the therapeutic antibody (Figure 2D).

Moreover, electrostatic interaction has been shown to be the other approach for conjugating delivered substances to magnetic NPs. For instance, Chertok et $\mathrm{al}^{23}$ have loaded $\beta$-galactosidase ( $\beta$-gal) onto heparin-coated iron-oxide NPs along with complex polyethyleneimine. The resulting nanocarrier exhibited capability in delivering a significant quantity of $\beta$-gal selectively into a brain tumor, revealed in a rat glioma model. While the previous method required $40-200 \mathrm{nmol} / \mathrm{kg}$ administration of the protein transduction domain peptide- $\beta$-gal conjugates to attain a detectable level of $\beta$-gal in the brain, this method facilitated $\beta$-gal accumulation in the brain tumor with a dose as low as only $4 \mathrm{nmol} / \mathrm{kg}$, a remarkable $10-50$-fold dose reduction. The methodology was claimed to be applicable to a wide variety of protein therapeutics.

The benefit provided by the synergistic effect of dual drugs and the development of codelivery of different drugs using the same vehicle has been exhibited. For instance, Singh el $\mathrm{al}^{24}$ prepared and characterized magnetic NP-embedded polylactide-co-glycolide matrices (PLGA-MNPs) as a dual drug-delivery and imaging system capable of encapsulating both hydrophilic (carboplatin) and hydrophobic (paclitaxel and rapamycin) drugs. The formulated PLGA-MNP nanocarrier showed a better contrast effect than commercial contrast agents as a result of higher $T 2$ relaxivity in connection to a blood circulation half-life of $\sim 47$ minutes in the rat model, furnishing the possibility of codelivery of single or multiple drugs toward active targeting (by means of herceptin-HER2 immunoreaction) with an improved synergistic therapeutic index.

Aside from the polymer-coated magnetic nanocarriers illustrated above, a number of nonpolymer-based 
biofunctionalizations have also been investigated. Magnetic NPs were functionalized with grafted moiety-enabling covalent coupling to drug, targeting agent, and chromophore for corresponding purposes. A previous study conducted in $2009^{25}$ demonstrated that superparamagnetic IONPs modified with a hydrophilic, biocompatible, and biodegradable coating of $N$-phosphonomethyl iminodiacetic acid, which was subsequently coupled, via an amine derivation, to desired functional molecules (such as folic acid, rhodamine $\mathrm{B}$ isothiocyanate, and methotrexate) endowed simultaneous targeting, imaging, and intracellular drug-delivery capabilities. Unique characteristics of this nanocarrier comprised a $\mathrm{pH}$-labile ester linkage (in connection to methotrexate) to facilitate drug release inside the acidic tumor endosomes, as well as magnetically guided drug delivery, resulting in the yield of enhanced T2-weighted MRI contrast. Similarly paclitaxel-loaded magnetic nanoparticles (pac-MNPs) were synthesized by Mitra et $\mathrm{al}^{26}$ whereby pac-MNPs were further functionalized with lectin by ethyl(dimethylaminopropyl) carbodiimide/ $N$-hydroxysuccinimide chemical conjugation, aiming to develop an active targeted nanotherapy strategy with higher cellular uptake and lower $\mathrm{IC}_{50}$. Both pac-MNPs and lectin-conjugated pac-MNPs were shown to exhibit a prolonged circulation time in serum, suggesting increased bioavailability and therapeutic index of paclitaxel in vivo.

Specifically, a nanocomposite probe consisting of superparamagnetic IONPs decorated with satellite CdS:Mn/ $\mathrm{ZnS}$ quantum dots has been developed. Quantum dots of the probe are further functionalized with STAT3 inhibitor, vitamin folate, and m-PEG, working as anticancer agent, targeting motif, and hydrophilic dispersing agent, respectively. Interestingly, the cellular uptake of the probe and subsequent drug release was indicated at the "on" state of quantum-dot luminescence upon the exposure of the probe to the cytosolic glutathione-containing environment (ie, intracellular), in contrast to the "off" state, at which luminescence was quenched as a result of conjugation with electron-rich ligands. The in vitro studies in folate receptor-overexpressed cancer cells validated the probe functionality, presented by MRI. ${ }^{27}$

With regard to the therapeutic option, a typical protocol in photodynamic therapy (PDT) involves administration of light-sensitive molecules (also called photosensitizers), which are subsequently activated with light at the desired wavelength and power. Upon activation, the photosensitizer molecules transfer the activated energy to surrounding oxygen, resulting in the production of reactive oxygen species (eg, singlet oxygen), which cause cell death. However, the drawbacks of photosensitizers include poor water solubility and inadequate selectivity, which limit clinical applications. To address this challenge, Huang et $\mathrm{al}^{28}$ rendered one of the second-generation photosensitizers, chlorine e6, covalently anchored on the surface of magnetic NPs with a silane coupling agent, resulting in a theranostic nanoparticle ( $20 \mathrm{~nm}$ in diameter) giving advantageous water solubility and biocompatibility, as well as noncytotoxicity and remarkable photodynamic efficacy. Such synthesized multifunctional nanocarriers were effective in simultaneous targeting by PDT in dual modality of NIR fluorescence and MRI of gastric cancer tissue in vivo.

On the other hand, the manipulation of NPs to raise the temperature of the body, whole or focused, to destroy a small area of cells or allow other types of cancer treatment to be more effective has been widely demonstrated. The formulation of the nanoparticles can crucially affect the efficacy of hyperthermia. Park et $\mathrm{al}^{29}$ described a novel synthesis and a systematic in vitro evaluation and application of magnetic iron cobalt nanoparticles (as a core) and a graphitic carbon (shell) for the targeted delivery of small interfering RNA (siRNA) to tumor cells with a concomitant hyperthermia-based therapy. This strategy cooperatively inhibited proliferation and induced apoptosis in tumor cells. Similar application of hyperthermia was also reported by Elsherbini et $\mathrm{al}^{30}$ where $\mathrm{Fe}_{3} \mathrm{O}_{4}$ NPs were utilized to simultaneously raise the temperature to $47^{\circ} \mathrm{C}$ under radiofrequency exposures at $25 \mathrm{~kW}$; at this point, the monitoring of the apoptotic cells presented dark signal intensity in the longitudinal relaxation time (T1)-weighted images, as investigated in Ehrlich tumors. In addition, synthesis of sugarcoated iron oxide NPs designed specifically as magnetic fluid hyperthermia heat mediators and negative contrast agent for MRI has also been described. ${ }^{31}$ The average size of iron oxide NPs was 4-35 nm, and the surface of which was coated with organic ligands bearing a phosphonate group and sugars (with rhamnose, mannose, or ribose moieties). These synthesized nanocarriers were able to be completely dispersed in water, resulting in the formation of colloids that were found to be stable over very long periods. Furthermore, such nanocarriers possessed advanced transverse relaxivity (three times larger than the best currently available commercial products) and large heat release upon application of radio-frequency electromagnetic radiation, with amplitude and frequency close to the human tolerance limit.

\section{Polymeric nanomaterials}

Polymeric micelles/conjugates have been emerging as a highly integrated theranostic nanoplatform for cancer diagnostics and therapy. Of numerous materials, the most 
successful strategy has been to modify nanocarriers with PEG, which leads to decreases in immunogenicity and antigenicity as well as increases in body-residence time and stability. Furthermore, PEG is capable of shielding the core of nanocarriers from degradation by steric hindrance, reducing kidney clearance by virtue of an increased hydrodynamic size of PEG-carrier conjugate, and increasing the solubility of nanocarriers as a result of its hydrophilicity.

There have been a great number of studies to reveal polymer-based theranostic agents by features of advantageous encapsulation properties of polymers. For instance, Guthi and coworkers $^{32}$ reported a successful development of prototype on a multifunctional polymeric micelle system in which superparamagnetic IONPs and DOX were encapsulated in the micelle core for MRI and therapeutic delivery, respectively, wherein the micelle surface was functionalized with a lung cancer-targeting peptide (LCP). Amphiphilic block copolymers, maleimide-terminated PEG-co-poly(D-, L-lactic acid) (Mal-PEG-PLA) and methoxy-terminated PEG-co-poly(D-, L-lactic acid) (MeO-PEG-PLA) were synthesized as the micelle core, taken with an incorporation of superparamagnetic IONPs, to exhibit superb $T 2$ relaxivity $\left(>400 \mathrm{Fe} \mathrm{mM}^{-1} \mathrm{~s}^{-1}\right)$ for ultrasensitive MR detection. LCPconjugated nanocarrier demonstrated significantly improved cell targeting and better cellular uptake in $\alpha_{\mathrm{v}} \beta_{6}$-expressing H2009 cells over scrambled peptide-encoded ones, or in $\alpha_{\mathrm{v}} \beta_{6}$-negative $\mathrm{H} 460$ cells.

The PEG-PLA micelles could also operate as a three-inone nanocontainer, encapsulating three poorly water-soluble drugs - paclitaxel, 17-allylamino-17-demethoxygeldanamycin, and rapamycin (PTX/17-AAG/RAPA) - for neoadjuvant cancer therapy (Figure 3A). ${ }^{33}$ In an LS180 human colon xenograft model, a single intravenous injection of such PEG- $b$-PLA micelles reduced tumor volume by 1.6-fold with $<10 \%$ body-weight change. Subsequent to the first intravenous injection, an injection of PEG-blockpoly( $\varepsilon$-caprolactone) (PEG- $b$-PCL) micelles to carry a carbocyanine dye showed a 2.1-fold higher NIR optical signal from excised solid tumors (versus a negative control) (Figure 3B), presumably due to a reduction in tumor cell density and interstitial tumor pressure. Also, the neoadjuvant therapeutics utilizing PTX/17-AAG/RAPA that contained
A

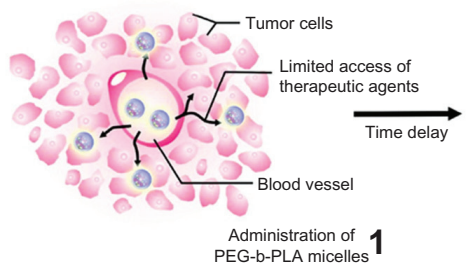

B

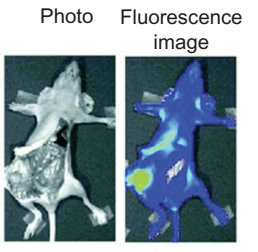

Vehicle

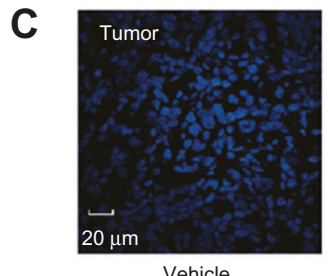

Vehicle

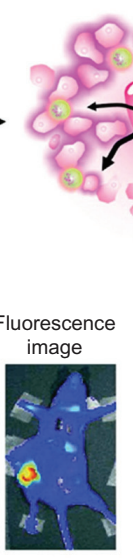

PTX
PTX
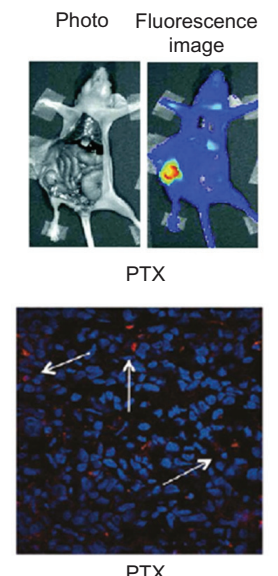

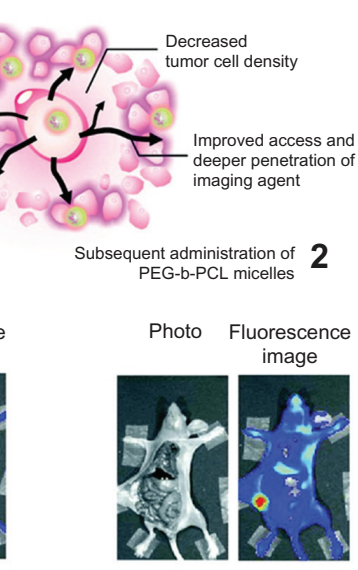

PTX/17-AAG/RAPA

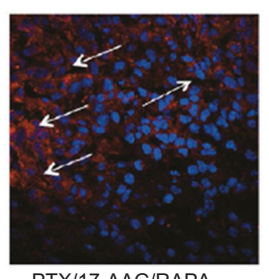

PTX/17-AAG/RAPA

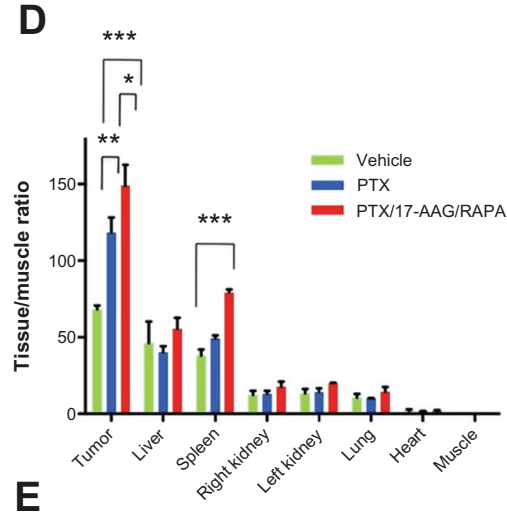

E

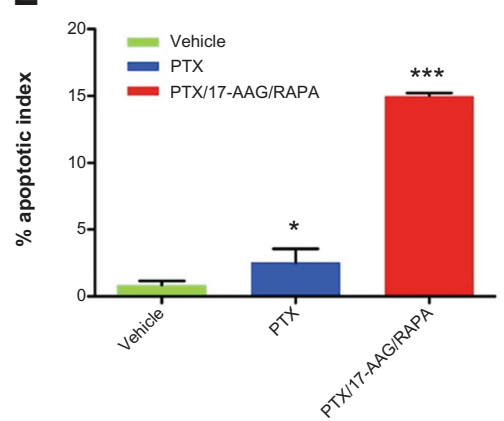

Figure 3 (A) Schematic illustration of tumor-primed delivery of NIR optical imaging agent (DiR) by a tandem of three-in-one PEG-b-PLA and PEG-b-PCL micelles; (B) wholebody NIR optical images of DiR (solid tumors in the right flank) after a midline incision in the abdomen; color alteration from blue to red indicates increased average radiant efficiency; (C) laser scanning confocal microscopic images of tumor tissue (60x magnification); DiR is shown in red, and nuclei of cells are in blue (DAPI); (D) summarized histograms of ex vivo NIR optical imaging of DiR after treatment with empty PEG-b-PLA micelles (vehicle), and micelle-encapsulated paclitaxel (PTX), or PTX/I7-AAG/RAPA, injected intravenously 48 hours before the injection of DiR-containing PEG-b-PCL micelles (mean $\pm \mathrm{SD} ; \mathrm{n}=3$; $* P<0.05$, **P $<0.0 \mathrm{I}$, ***P $<0.000 \mathrm{I}$ ); (E) apoptosis index recorded in immunohistochemical analysis of apoptosis (mean $\pm S D ; n=3 ; * P<0.05$, *** $P<0.0001$ ).

Notes: (B and C) Ex vivo and in vitro tests treated with vehicle control (left), PEG-b-PLA micelles carrying PTX (middle), and three-in-one PEG-b-PLA micelles carrying PTX/I7-AAG/RAPA (right); Reprinted with permission from Cho H, Kwon GS. Polymeric micelles for neoadjuvant cancer therapy and tumor-primed optical imaging. ACS Nano. Copyright @ 201 I American Chemical Society. ${ }^{33}$

Abbreviations: NIR, near infrared; PEG-b-PLA, polyethylene glycol polylactic acid; PCL, polycaprolactone. 
nanocarrier exhibited improved tumor-to-muscle ratio and a high apoptosis index of cancer cells (Figure 3D and E).

Given varied monomers that have been copolymerized with poly(D-, L-lactic acid) to form multifunctional polymeric carriers, the principal capability of poly(D-, L-lactic acid) is to yield a hydrophobic environment to encapsulate hydrophobic drugs (eg, DOX and paclitaxel) more efficiently. The work presented by Lu et $\mathrm{al}^{34}$ illustrated a self-assembly of methoxyl/functionalized-PEG-PLA diblock copolymers, grafted with poly( $N$-vinylimidazole- $\mathrm{Co}-\mathrm{N}$-vinylpyrrolidone)g-poly(D-, L-lactide), leading to the formation of carrier for DOX delivery. Specifically a $\mathrm{pH}$-sensitive structure of imidazole served as the triggering moiety (ie, a folic acid/ folate receptor-targeted binding, followed by the release of DOX in acidic surroundings). Imaging of ${ }^{123}$ I-labeled NPs by single-photon emission computed tomography was conducted to ensure intratumoral accumulation. In vivo tumor growth inhibition showed that the nanocarriers exhibited excellent antitumor activity and a high rate of apoptosis in cancer cells, and furthermore, no heart, liver, or kidney damage was found substantially by DOX or polymeric materials through the 80-day treatment course.

Similarly, a scenario utilizing "amphiphilic" polymers as nanovesicles was presented by Yang et $\mathrm{al}^{35}$ and $\mathrm{Xu}$ et al. ${ }^{36}$ The former synthesized a triblock copolymer $\left(\mathrm{PEG}_{114^{-}}\right.$ poly(glutamate)-PEG ${ }_{46}$ ), of which DOX was conjugated to the polyglutamate segment via a $\mathrm{pH}$-sensitive hydrazone bond. It was observed that the long PEG segments were mostly segregated into outer hydrophilic PEG layers of the vesicles, thereby providing active tumor targeting via folate. In contrast, the short PEG segments were segregated into the inner hydrophilic PEG layer of the vesicles, making it possible to cross-link with the inner PEG layer via acrylate groups for better in vivo stability. In addition, hydrophilic superparamagnetic IONPs were encapsulated into the aqueous core of the stable vesicles, allowing for ultrasensitive MRI detection. Such IONPs/DOX-loaded vesicles demonstrated a much higher transverse relaxation rate $(r 2)$ than Feridex, (AMAG. Pharmaceuticals, Cambridge, MA, USA) a commercially available superparamagnetic iron oxide (SPIO)-based $T 2$ contrast agent, attributed to the high superparamagnetic IONP loading level and the clustering effect in the aqueous core of the vesicles. It was concluded by the authors ${ }^{35}$ that folic acid-conjugated vesicles exhibited higher cellular uptake than folic acid-free vesicles, based on the results obtained from flow cytometry and confocal laser scanning microscopy. Correspondingly, the latter study described a unique encapsulation of both up-conversion and iron oxide NPs using an amphiphilic block copolymer, poly(styrene-block-allyl alcohol) $\left(\mathrm{PS}_{16}\right.$-b-PAA $\left._{10}\right)$, via a microemulsion method. This nanocarrier rendered dual modalities of upconversion luminescence and MRI, and once squaraine dye for down-conversion fluorescence was incorporated, a nanocomposite combining triple-modal imaging was created.

Chitosan has also been used as a polymeric material for developing theranostic nanocarriers. $\mathrm{Na}$ et $\mathrm{al}^{37}$ designed theranostic chitosan NPs carrying Cy5.5 for live imaging and paclitaxel for cancer treatment. In these investigations, glycol chitosan NPs were further modified with hydrophobic $5 \beta$-cholanic acid to confer the localized region of nanocarrier for the encapsulation of hydrophobic paclitaxel. It was suggested that the superior tumor-targeting ability in various tumor models originated from such key factors as in vitro/in vivo stability, deformability, and rapid uptake by target tumor cells. Pursuant to the results from real-time monitoring of chitosan NPs in tumor vasculature, the tumortargeting ability was enabled by long circulation and penetration through fenestrate angiogenic vessels in tumor tissue.

Polymeric materials could also be employed as the "core" of theranostic nanocarriers along with attached "shell" for diverse purposes (eg, improvement of hydrophilicity, encapsulation of drug, and coupling of magnetic agent and dye for imaging). Specifically, indocyanine green, an FDAapproved photosensitizer, and PEG of various molecular weights were attached to poly(propargyl acrylate) colloid core through a copper-catalyzed azide/alkyne cycloaddition. It was found that the inclusion of PEG with indocyanine green onto the particle surface resulted in a synergistic enhancement of the fluorescence intensity, with increasing molecular weight of PEGs amplifying the response. Preliminary PDT studies carried out in the albumin-overexpressed human liver carcinoma cells (HepG2) indicated that a minor exposure of 780-nm radiation resulted in a statistically significant reduction of cell growth. ${ }^{38}$

A number of investigations have utilized poly(amino acid) to generate theranostic nanocarriers. Interestingly, Santra and Pere $\mathrm{z}^{39}$ presented a synthetic strategy - a single-step approach for synthesis of an $\mathrm{N}$-alkylated amino acid as an AB-type functional monomer - to facilitate the synthesis of a poly(amino acid) without using the laborious protection-deprotection methods. Notably, the synthesized amino acid-based polymer with butyl pendants enhanced its solubility in common organic solvents and facilitated the construction of hydrophobic nanocavities for the effective encapsulation of hydrophobic DiI dye and the therapeutic drug Taxol (Sigma, St. Louis, MI, USA). 
In addition, cell cytotoxicity studies confirmed the low toxicity of the polymeric nanoparticles, and the Taxol-encapsulated NPs only exerted cytotoxicity upon internalization into folate receptor-overexpressed cancer cells.

Moreover, Yang and coworkers ${ }^{40}$ developed a poly(aspartic acid) nanocarrier that was synthesized via acid-catalyzed polycondensation of L-aspartic acid using phosphoric acid as the catalyst, and subsequently octadecylamine was added to form octadecyl-grafted poly(aspartic acid). Then iron oxide nanocrystals and the DOX drug were loaded in poly(aspartic acid) NPs through an emulsion method. Such a nanocarrier shows a twofold higher $r 2$ value relative to that of a commercial product, and DOX was successfully delivered into cancer cells. Similar work incorporating gadolinium (Gd)-diethylenetriaminepentaacetic acid as contrast agent and (1,2-diaminocyclohexane)platinum(II) as potent anticancer drug, was performed, ${ }^{41}$ where a coreshell polymeric micelle comprised of PEG- $b$-poly(glutamic acid) was synthesized for cancer theranostics. The results indicated that stronger tumor contrast enhancement (24 times increase on longitudinal relaxation time $r 1$ ) was achieved by the micelles compared to the free Gd chelates, which was ascribable to the flexibility reduction per Gd molecule after binding with polymers or proteins.

Hyaluronic acid (HA) has also been confirmed as a targeting substance due to its capability of binding specifically with various cancer cells that overexpressed CD44, an HA receptor. It was therefore, by this feature, shaped in nanoparticle fashion (HA-NPs) and effectively accumulated in the tumor site. However, a significant portion of HA-NPs could also be found in the liver, which may limit their practical application for tumor therapy and diagnosis. To address this challenge, Choi and coworkers have demonstrated that appropriate PEGylation of HA-NPs may result in reduced uptake in the liver, prolonged blood circulation, and improved tumor targetability. ${ }^{42}$

PLGA, a biodegradable polymer, has been employed to design theranostic nanocarriers. In work by Pan et $\mathrm{al}^{43} \mathrm{D}-\alpha-$ tocopheryl PEG 1000 succinate-COOH (TPGS-COOH) was copolymered to ensure high encapsulation efficiency, desired drug-release profile, and high cellular adhesion. More importantly, it was observed that the targeting effects could be controlled by tuning the ratio of PLGA and TPGS$\mathrm{COOH}$. In connection to an encapsulation of quantum dots as a model-imaging agent and DOX as a model anticancer drug, it was revealed that NPs with folate conjugation exhibited higher cellular uptake compared with those without folate conjugation in a cell model of MCF-7, yet not for NIH 3T3 cells.
Distinct from most studies regarding harnessing drug release as the therapeutic strategy, an interesting study by Kojima et al demonstrated the preparation of AuNP, loaded in a PEGylated dendrimers matrix, as imaging and therapeutic agents simultaneously. The grown AuNP enabled not only efficient $\mathrm{CT}$ imaging but also photothermogenic properties (ie, AuNP is capable of attacking the tissue upon the activation of light-induced heat), thereby holding potential to be a photothermal therapeutic tool. ${ }^{44}$

\section{Silica-based nanomaterials}

Unlike many other nanomaterials, of which size-dependent properties are commonly observed as their size approaches the nanoscale and as the percentage of atoms at the surface of a material becomes significant, silica-based nanoparticles (SiNPs) have constant physical properties similar to those of bulk material, except the total surface area increases as the size decreases. In addition to the higher surface area, what really makes $\mathrm{SiO}_{2}$ NPs prevail in nanobiomedicine is their well-defined tunable nanostructures and well-established siloxane chemistry, which allow us to fabricate effectively the desired functionalized surface for diagnostic and therapeutic applications.

He et al described the development of a bifunctional NP-based carrier for simultaneous in vivo imaging and PDT by encapsulating methylene blue (MB) in the phosphonate-terminated silica matrix (PSiNPs). ${ }^{45} \mathrm{MB}$ served as the photosensitizer, was encapsulated inside the PSiNPs, and was further protected from reduction by diaphorse. A sufficient dose of irradiation to the MB-encapsulated PSiNPs under a light of $635 \mathrm{~nm}$ resulted in the production of singlet oxygen that led to photodynamic damage to Hela cells. Moreover, it was also confirmed that NIR luminescence could be emitted by the MB-encapuslated PSiNPs, providing image guidance for site-specific PDT.

Mesoporous silica nanoparticles (MSNs) are prospective candidates for well-developed theranostic NPs. The ultrahigh surface area of MSNs provides a larger extent of spaces to be functionalized with desired ligands. In a study by Zhang et al, a multifunctional SiNP containing a nonporous dye-doped silica core and a mesoporous silica shell enclosing photosensitizer molecules, called hematoporphyrin, was designed and synthesized. ${ }^{45}$ The mesoporous silica nanovehicle acted as not only a carrier for the photosensitizers but also a nanoreactor to facilitate the photo-oxidation reaction. Furthermore, the efficiency of photooxidation of the hematoporphyrin was significantly improved. Likewise, a study by Cheng et $\mathrm{al}^{47}$ reported 
development of trifunctionalized MSNs for theranostic agent utility that combined imaging, targeting, and therapy in one single-particle platform. This theranostic platform with cRGDyK peptides modified onto the exterior surface of MSNs exhibited excellent targeting of the overexpressed $\alpha_{\mathrm{v}} \beta_{3}$ integrins of U87-MG human glioblastoma cells and minimal collateral damage, but highly potent therapeutic effects as well in vitro.

The large surface area and porous interior of MSNs enable them to provide reservoirs for storing, delivering, and releasing an adequate supply of drugs. Lu et al described investigations on biocompatibility of fluorescent MSNs (FMSNs) and biodistribution in mice with established human cancer xenograft using in vivo imaging, fluorescence microscopy, and mass spectrometry. In addition, the ability of FMSNs to deliver anticancer drugs into human xenograft in mice and to suppress tumor growth was confirmed. ${ }^{48}$ The authors claimed that their FMSNs were well tolerated in mice, preferentially accumulated in tumors and an elevated accumulation of particles in folate receptor-overexpressing cells, and more cell death-inducing effects were observed with folic acid-conjugated-FMSNs than nontargeting FMSNs.

The outer surface of MSNs can be functionalized with other materials to improve their applicability in theranostics. Pursuant to the results obtained by Lee et al ${ }^{49}$ a highly versatile nanocomposite nanoparticle was designed and prepared by decorating the surface of mesoporous dye-doped SiNP with multiple magnetite nanocrystals, giving the diagnostic capacity of MRI to MSNs. In a recent study by Meng et al, a new approach was investigated to overcome multiple drug resistance in cancer cells by means of the codelivery of siRNA that silenced the expression of efflux transporter together with an appropriate anticancer drug for drug-resistant cancer cells. ${ }^{50}$ It was confirmed by the authors that polyethyleneimine-modified MSNs loaded with P-glycoprotein (Pgp) siRNA and DOX were able to demonstrate an effective delivery of a chemotherapeutic agent, DOX, as well as Pgp siRNA to KB-V1 cells to accomplish cell death in an additive or synergistic fashion.

\section{Carbon nanomaterials}

Based on their bonding structures $\left(\mathrm{sp}^{2}\right.$ or $\left.\mathrm{sp}^{3}\right)$, carbon nanomaterials can be classified into zero-dimensional fullerene, carbon dot, nanodiamond, one-dimensional carbon nanotube, and two-dimensional graphene. Each of them possesses exceptional physical and chemical properties. For instance, by virtue of their optoelectronic properties, carbon dot and nanodiamond are able to display natural fluorescence emission. Graphene and carbon nanotube, on the other hand, exhibit unique mechanical, electrical, thermal, and biological properties for a variety of applications, including nanomedicine. Robinson et al were the first to demonstrate dual application in NIR imaging and PTT using PEGylated phospholipid functionalized single-walled carbon nanotube (SWNT). ${ }^{51}$

SWNT is one potential candidate for us as a theranostic agent due to its strong optical absorbance in the NIR region, which can not only emit photoluminescence in the $1.0-1.4 \mu \mathrm{m}$ region for in vivo tumor imaging but also act as an efficient NIR absorber and heater (SWNT increases the local temperature of tumor as high as $60^{\circ} \mathrm{C}$ under 5 minutes of 808-nm laser irradiation) for photothermal ablation of tumors with low injected dose. ${ }^{52}$ Compared with the treatment using AuNRs, highly effective tumor elimination with SWNTs was attained at 10-fold lower injected doses and lower irradiation powers.

Likewise, graphene has also been demonstrated as a photothermal transducer due to its higher optical absorbance in the NIR region. According to an observation made by Yang et $\mathrm{al}^{53}$ a surprisingly high tumor uptake of nanographene sheet (NGS) (Figure 4A) was found in several xenograft tumor-bearing mouse models (Figure 4C). Compared with PEGylated carbon nanotube, PEGylated NGS showed superior efficient tumor passive targeting and less retention in reticuloendothelial systems. ${ }^{53}$ Such PEGylated NGS was further used for in vivo PTT, revealing that an ultraefficient tumor ablation after intravenous administration of NGS and low-power NIR laser irradiation on the tumor could be reached (Figure 4B, D, and E). Furthermore, no obvious side effect of PEGylated NGS was noticed in this study for the injected mice by histology, blood chemistry, and complete blood panel analysis.

\section{Composite nanomaterials}

Multifunctionalities (including targeting, imaging, and therapy) are indispensable in the design of theranostic agents that are expected to overcome limitations associated with conventional cancer diagnosis and therapy. However, most types of nanomaterials possess one unique function that can possibly be used for either therapeutic or diagnostic applications.

Hybrid nanomaterials, consisting of different nanomaterials, have recently been investigated as promising platforms for diagnostic, imaging, and therapeutic applications. To exclude composite from polymeric nanocarriers illustrated previously, nanomaterial composed of 
A

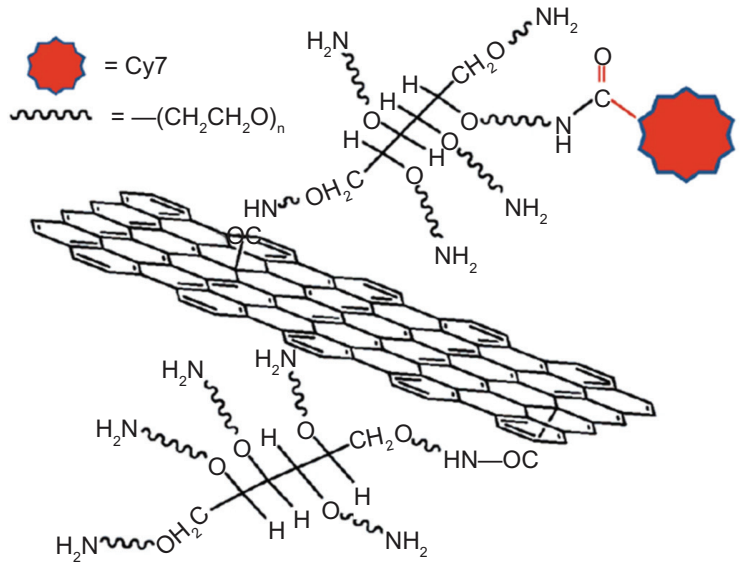

C

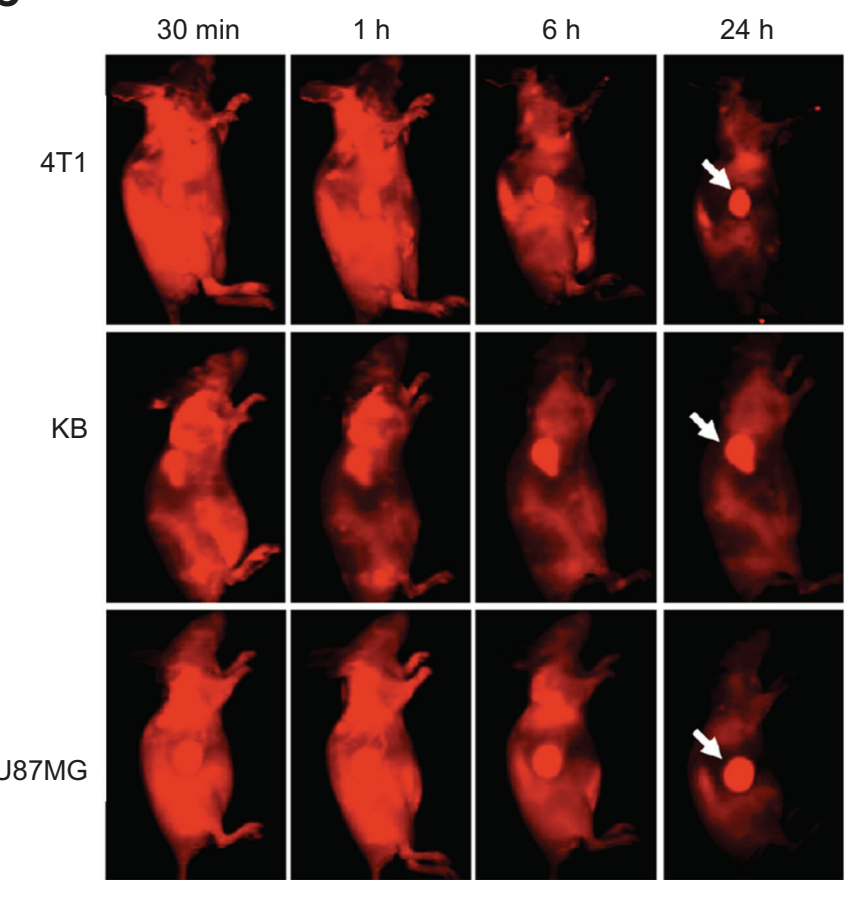

B

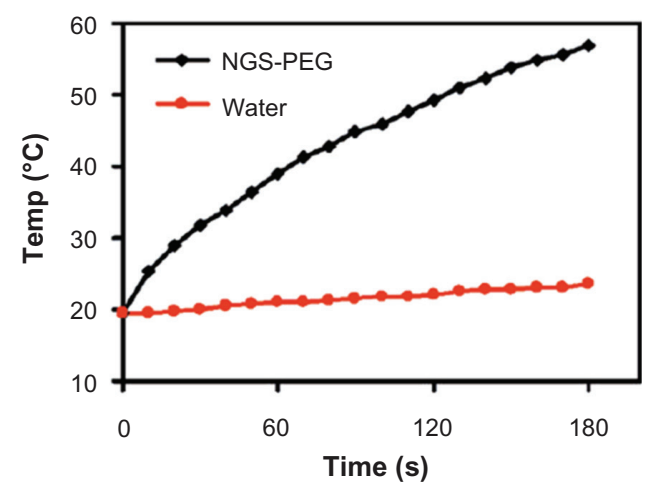

D

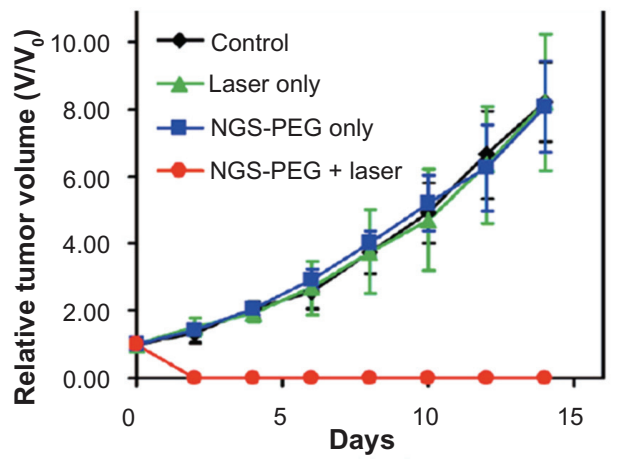

E

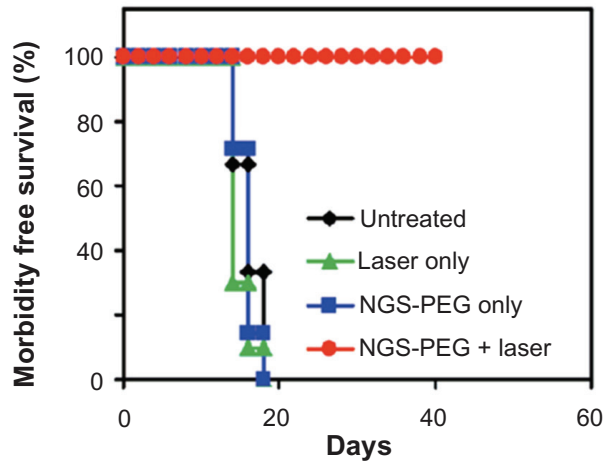

Figure 4 (A) Scheme of an NGS with PEG functionalization and labeled by Cy7; (B) temperature-change curves of NGS-PEG solution and water exposed to 808-nm laser at power density of $2 \mathrm{~W} / \mathrm{cm}^{2}$; rapid rise in temperature was noted for the NGS-PEG solution, in marked contrast to the water temperature, which showed little change during the laser irradiation; (C) spectrally unmixed in vivo fluorescence images of $4 \mathrm{TI}$ tumor bearing Balb/c mice, KB, and U87MG tumor-bearing nude mice at different time points post-injection of NGS-PEG-Cy7; high tumor uptake of NGS-PEG-Cy7 was observed for all three tumor models; (D) tumor-growth curves of different groups after treatment, tumor volumes normalized to initial sizes; while injection of NGS-PEG by itself or laser irradiation on uninjected mice did not affect tumor growth, tumors in the treated group were completely eliminated after NGS-PEG injection and following NIR laser irradiation; (E) survival curves of mice bearing 4TI tumor after various treatments; NGS-PEG-injected mice after photothermal therapy survived over 40 days without a single death.

Reprinted with permission from Yang K, Zhang S, Zhang G, Sun X, Lee ST, Liu Z. Graphene in mice: ultrahigh in vivo tumor uptake and efficient photothermal therapy. Nano Lett. Copyright (C) 2010 American Chemical Society. ${ }^{53}$

Abbreviations: NGS, nanographene sheet; PEG, polyethylene glycol; NIR, near infrared.

more than two nanomaterials without polymer encapsulation are selected here as composite nanomaterials. In 2009, Ma et al reported that a photothermal effect induced by 20-50-nm stable NIR-active, superparamagnetic nanoroses formed by gold-coated IONPs,${ }^{54}$ allowed them to achieve photothermal therapy. They also found that high uptake of the nanoroses by macrophages was further improved by dextran coating, producing intense NIR contrast in dark-field and hyperspectral microscopy, in both in vitro and in vivo rabbit models of atherosclerosis. The authors claimed that the obtained small nanoroses with multifunctionality in optical, magnetic, and therapeutic applications, offer wide opportunities for targeted cellular imaging, therapy, and combined imaging and therapy.

To combine PTT and MRI, a similar combination of nanomaterials was also described by Melancon et $\mathrm{al}^{55}$ in which 
SPIO was used as core, followed by the coating of amorphous silica shell and the growing of a monolayer of gold on the shell to form the SPIO@AuNS nanocomposite.The SPIO@ AuNS possessed a high NIR absorption in the NIR region (650-900 nm), which was important for photothermal ablation using an NIR laser. The temperature increases were manipulated as a function of the concentration of SPIO@AuNS, laser power, and irradiation time. The MRI ability and photothermal effect were confirmed to be feasible on tumor-bearing mouse through MRI and MR temperature imaging.

Instead of using amorphous silica, mesoporous silica was utilized by Ma et al for surface modification of AuNR-linked magnetic nanoparticle, leading to the production of a novel photothermal sensitizer (AuNR-MMSNEs) (Figure 5A). ${ }^{56}$ The magnetic core, AuNR, and mesostructure of the silica shell provided the functionalities in MRI, PTT, and drugloading capability, respectively. This hybrid AuNR-MMSNE integrated chemotherapy, MRI (Figure 5B), PTT (Figure 5D), IR thermal, and optical imaging into one system. The release profile of the encapsulated drug (DOX) indicated half of the loaded drug could be released within 5 hours under acidic conditions ( $\mathrm{pH} 5.5$ ) (Figure 5C). In addition, a synergistic effect on tumor-inhibition rate was also observed through the combination of PTT and chemotherapy (Figure 5E).
A

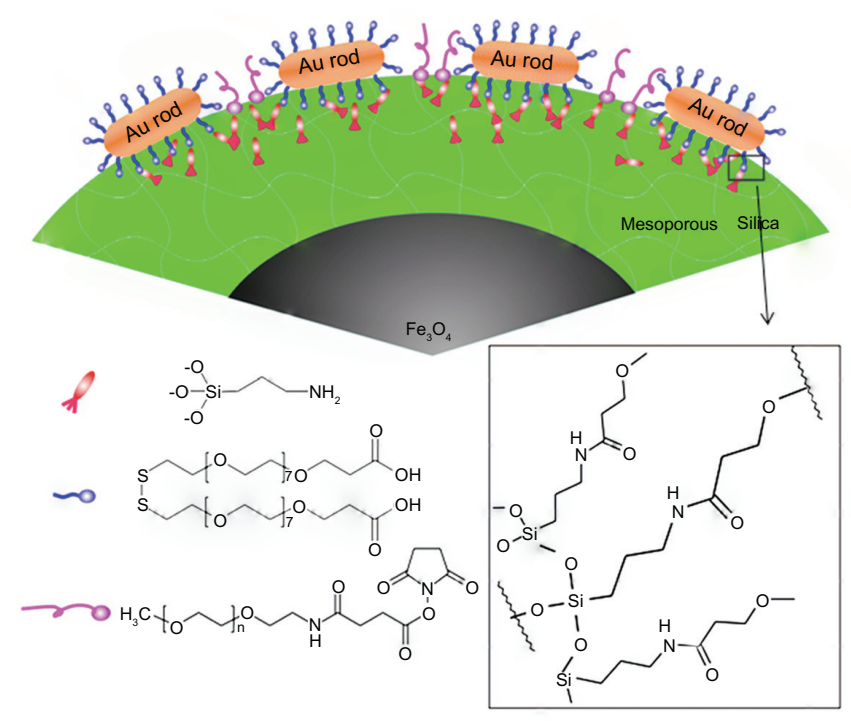

B
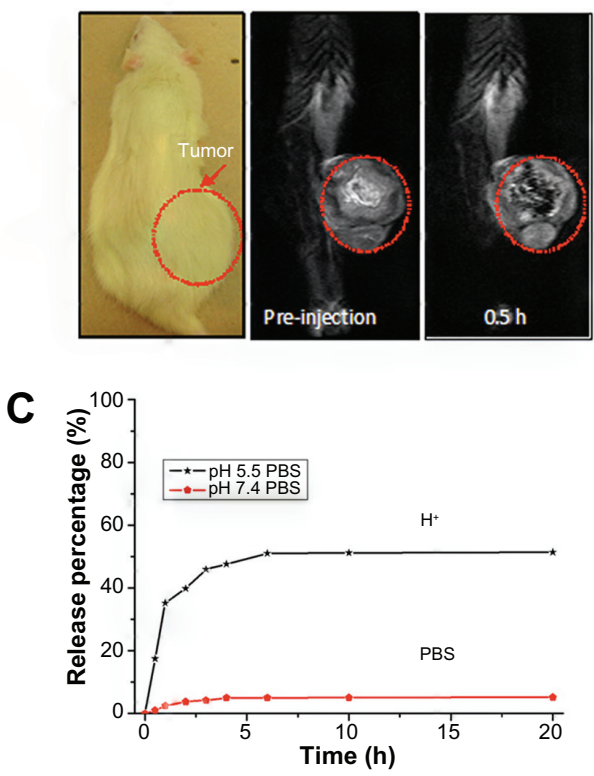

D

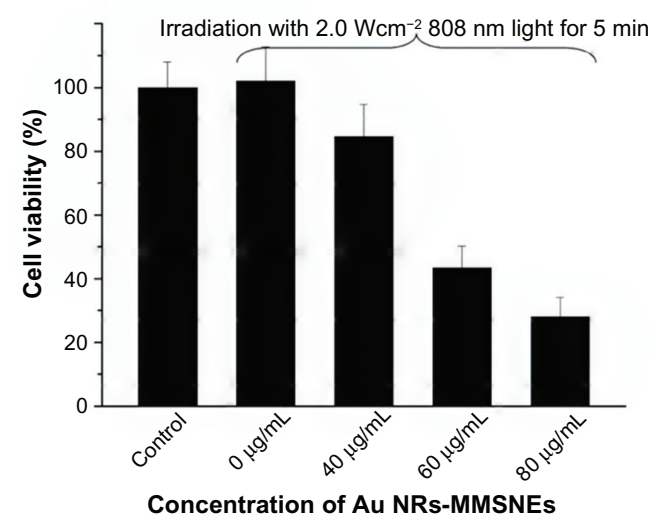

E

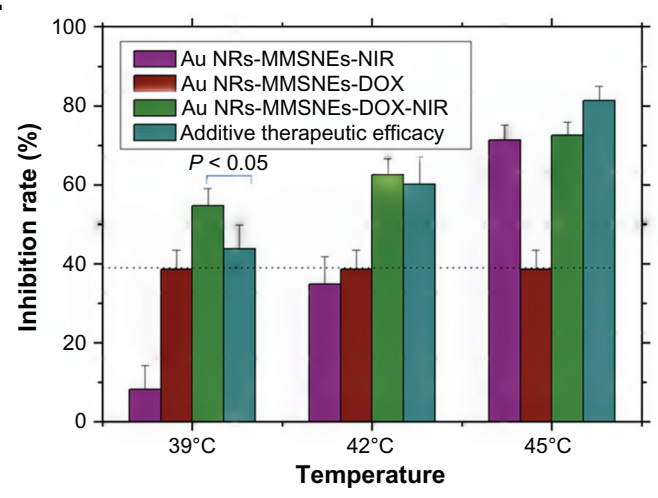

Figure 5 (A) Schematic representation of AuNR-MMSNEs: numbers of AuNRs being capped on the outer surface of a magnetite core/mesoporous silica shell; (B) In vivo magnetic resonance imaging of a mouse before and after intratumoral injection of AuNR-MMSNEs; (C) in vitro release profiles of Au NR-MMSNE-DOX using dialysis membrane against phosphate-buffered saline solution at pH 7.4 and 5.5; (D) cell viabilities for MCF-7 cells treated with different concentrations of AuNR-MMSNEs after incubation for 24 hours, after laser exposure for 5 minutes; (E) comparison of inhibition rates for MCF-7 cells treated by AuNR-MMSNE-NIR (purple), AuNR-MMSNEDOX (red), and AuNR-MMSNE-DOX-NIR (green); for photothermal treatment, the media were under 808-nm laser irradiation for 5 minutes at different power intensities, corresponding to maximum temperature increases to $39^{\circ} \mathrm{C}, 42^{\circ} \mathrm{C}$ and $45^{\circ} \mathrm{C}$.

Reprinted from Biomaterials. 20I2;33(3):989-998. Ma M, Chen H, Chen Y, et al. Au capped magnetic core/mesoporous silica shell nanoparticles for combined photothermo-/ chemo-therapy and multimodal imaging (C) 2012 with permission from Elsevier. ${ }^{56}$

Abbreviations: AuNR-MMSNEs, gold nanorod-capped magnetic core/mesoporous silica shell nanoellipsoids; DOX, doxorubicin; NIR, near infrared. 
Loading a sufficient amount of drug onto/inside nanoparticles is important for an ideal carrier with therapeutic function. Mesoporous silica nanostructures, known to have larger pore volume, tunable pore size, easy surface modification, and great biocompatibility are therefore suitable for being developed as a drug reservoir for hybrid nanomaterials. However, strategies must be established to prevent silica-based nanomaterial from self-aggregation and nonspecific binding with protein under physiological condition. Our group previously described the design of a hybrid nanoshuttle consisting of phospholipid monolayer and MSNs,${ }^{57}$ and we found that the lipid layer was capable of efficiently preventing self-aggregation and nonspecific protein binding in the physiological fluids or salt-containing environment. Moreover, the targeting function of hybrid nanoshuttles toward the folate receptor-overexpressed Hela cells was achieved by incorporating folic acid-derived lipid, leading to the formation of folic acid-anchored, phospholipidcapped mesoporous silica nanoshuttles. We believe that the surface modification of phospholipids for inorganic or organic nanoparticles constructs a much more biocompatible platform for the development of theranostic nanomedicine, combining both therapeutic and diagnostic capabilities in one dose.

\section{Other nanomaterials}

In addition to the conventional nanomaterials (AuNPs, SPIO, polymeric NPs, SINPs), there has been increasing attention in employing upper-conversion nanoparticles (UCNPs) for theranostic applications. UCNPs are lanthanidedoped nanocrystals that emit higher light energy (visible) upon excitation by lower light energy (ie, $978 \mathrm{~nm}$ ) via an upconversion process. Electromagnetic waves in the NIR region are known to be relatively transparent in biological tissues. Therefore, combining UCNPs with photosensitizer may settle the major problem for PDT; that is, photosensitizer itself can only be excited by visible light that shows poor penetration ability in biological tissue. By encapsulating photosensitizer in the polymer modified on UCNP, PDT can be executed under NIR irradiation, in which biological tissues may have minimal light absorption. ${ }^{58,59}$ Through combination with other nanomaterials, UCNP should be multifunctional in both imaging and therapy. Cheng et al revealed a multifunctional probe by adsorbing IONPs onto the surface of an $\mathrm{NaYF}_{4}$-based UCNP, followed by the formation of a thin gold shell via seed-induced reduction growth. ${ }^{60}$ Such multifunctional up-conversion nanoprobes were demonstrated to be feasible in PTT and in vivo imaging executed by either MRI or NIR irradiation.
Quantum dots (QDs) are semiconductor nanocrystals that exhibit stable and bright fluorescence. By tuning particle size and composition, their optical properties can be adjusted. Thereby, CdSe, CdTe, InAs, and ZnSe have been utilized to synthesize QDs in varied sizes, resulting in the production of semiconductor nanoparticles that span the full visible and NIR spectrum. However, QD-based theranostic carrier has been relatively less investigated, principally owing to innate toxicity and instability. To confer QDs' higher solubility and stability, thiolated ligands were employed to couple onto the exterior of the QDs (or the $\mathrm{ZnS}$ shell) via a disulfide linkage. ${ }^{61}$ Such linkage, nevertheless, can be degraded by the intracellular biothiols, thereby resulting in compromised functionality. Zhu et $\mathrm{l}^{62}$ have demonstrated that such monolayer stability is correlated with both particle size of QD and monolayer structure, and with appropriate management of these two factors, the intracellular stability of QDs can be greatly enhanced.

Taking advantage of the excellent optical property of QDs for imaging purposes in connection with increased biocompatibility and stability, near-IR QD-loaded micelles have been investigated. ${ }^{63}$ 10,12-pentacosadiynoic acid (PCDA) was chosen as the hydrophobic moiety-forming element to facilitate drug encapsulation, and due to its UVresponsive polymerization capability, a strong outer shell via cross-linking was formed upon UV irradiation. Such lipid-conjugated QDs not only possessed reduced toxicity but also attained improved tumor cell uptake and retention. The intermicellar cross-linking polymerization of PEG-PCDA and PCDA-herceptin conjugates significantly stabilized the QDs and became applicable to biomedical imaging and therapy, along with a targeting towards cancer cells that overexpressed HER-2 receptor. Although the results collected from the animal studies suggested that near-IR QD-loaded micelles have enhanced antitumor activity (yielding 77.3\% reduction in tumor volume) and selective toxicity, further investigations are required to clarify the mechanism of the antitumor activity, thereby evidencing a superior therapeutic agent of near-IR QD-loaded micelles.

Silver NPs (AgNPs) were utilized as imaging-contrast agents in either core or shell fashions. For instance, porous silver coating was built around silica cores $(180-520 \mathrm{~nm})$ to enable a core-shell nanoplatform exhibiting high and broad optical extinction at NIR wavelengths, allowing it to be used in photoacoustic imaging. ${ }^{64}$ Such a silver nanosystem was detected at least $1 \mathrm{~cm}$ deep in ex vivo pancreatic tissue using combined photoacoustic and ultrasound imaging, and furthermore, the photoacoustic signal increased 
linearly with larger nanoparticle concentration. While the nanosystem has been demonstrated as a potential contrast agent for image-guided therapy, therapeutic molecules are not encapsulated in the silica yet. In a distinct manner, $\mathrm{Wu}$ et $\mathrm{al}^{65}$ developed a core shell-structured hybrid nanogel (40-80 nm) composed of AgNP as core and $\mathrm{pH}$-responsive gel of poly( $N$-isopropylacrylamide-co-acrylic acid) (p[NIPAM-co-AA] ) as shell. While the p(NIPAM-co-AA) gel shell served as a drug carrier with high loading capacity of hydrophobic dipyridamole drug, the AgNP core acted as an optical identification code. The shrinkage of $p$ (NIPAMco-AA) gel shell in acidic $\mathrm{pH}$ not only manipulated the optical properties of the core and resulted in an increase of the UV-Vis absorption intensity and blue shift but also changed the mesh size of the gel shell, enabling a controllable release. Moreover, it was revealed that the nanogel was able to conquer cellular barriers to enter the intracellular section and light up the mouse melanoma B16F10 cells. However, the interaction between nanogel and cells was somewhat altered by the surface property and concentration of the injected/applied (in vitro) hybrid nanogels.

\section{Conclusion and perspectives}

This paper provides an insight into recent efforts in this evolving field, in which we have highlighted theranostic nanoplatforms cored with gold, magnetic, polymer, composite, silica, carbon, and QDs.

Despite notable progress, there remains no nanotheranostic agent/particle that is sufficiently sophisticated to meet clinical standards. Most nanotheranostic platforms possess their own promises and advantages, but suffer from some weaknesses, with challenges to overcome, such as the cost of gold NPs, insufficient sensitivity of IONP acting as MRI contrast agent, complexity of composite carrier, the large size of silica NPs, and nonbiodegradable property of carbon materials. These issues therefore necessitate substantial efforts to utilize particular solutions accordingly.

Towards the ultimate goal of theranostics meeting the demand of personalized medicine, several perspectives can be identified: nanotheranostic platforms harnessing great capability allowing multiple modalities, either therapeutic or diagnostic, and furthermore both, is one of the main areas offering an all-in-one nanosystem with comprehensive features. However, the increasing complexity of the system, safety, and stability of nanoparticles will be highlighted along with further exploration of knowledge addressing long-term characterizations of nanomaterials. To this end, substantial in vivo examinations are of significance and highly required. In addition, monitoring the therapy and follow-up responses by utilizing fluorescent dyes and QDs has been realized at cellular level; this nevertheless suffers critical difficulty in body imaging. Further development is needed to permit high-resolution imaging and to mitigate the background fluorescence and photoleaching. On the other hand, the employment of NIR dye, offering a way to obtain high resolution and reproducible images, may be an appealing alternative approach for biomedical imaging.

The emergence of nanotechnology has offered a great opportunity to promote the design and development of innovative nanotheranostics. From this perspective, the detection modality has promise to be performed not only before or after but also throughout the treatment regimen. After comprehensive evaluations on cytotoxicity, genotoxicity, and immunotoxicity of prospective nanotheranostics are finished, and cost-effectiveness, accessible testing systems are available, the introduction of nanotheranostics into routine health care may thus become plausible as an important element of personalized and predictive medicine.

\section{Acknowledgments}

The authors are grateful to the National Science Council in Taiwan, under grants 98-2113-M-007-013-MY3, 100-2811M-002-076, 100-2113-M-002-016-MY2, and 100-2627-M006-006.

\section{Disclosure}

The authors report no conflicts of interest in this work.

\section{References}

1. Funkhouser J. Reinventing pharma: the theranostic revolution. Curr Drug Discov. 2002;2:17-19.

2. Park K, Lee S, Kang E, Kim K, Choi K, Kwon IC. New generation of multifunctional nanoparticles for cancer imaging and therapy. Adv Funct Mater. 2009;19(10):1553-1566.

3. Cai W, Chen X, Nanoplatforms for targeted molecular imaging in living subjects. Small. 2007;3(11):1840-1854.

4. Zhang Z, Jia J, Lai Y, Ma Y, Weng, J, Sun, L. Conjugating folic acid to gold nanoparticles through glutathione for targeting and detecting cancer cells. Bioorg Med Chem. 2010;18(15):5528-5534.

5. Heo DN, Yang DH, Moon HJ, et al. Gold nanoparticles surfacefunctionalized with paclitaxel drug and biotin receptor as theranostic agents for cancer therapy. Biomaterials. 2012;33(3):856-866.

6. Kim D, Kim JW, Jeong YY, Jon S. Antibiofouling polymer coated gold@ iron oxide nanoparticle (GION) as a dual contrast agent for CT and MRI Bull Korean Chem Soc. 2009;30(8):1855-1857.

7. Kim D, Jeong YY, Jon S. A drug-loaded aptamer-gold nanoparticle bioconjugate for combined $\mathrm{CT}$ imaging and therapy of prostate cancer ACS Nano. 2010;4(7):3689-3696.

8. Huang P, Bao L, Zhang C, et al. Folic acid-conjugated silica-modified gold nanorods for X-ray/CT imaging-guided dual-mode radiation and photo-thermal therapy. Biomaterials. 2011;32(36):9796-9809. 
9. Lukianova-Hleb EY, Hanna EY, Hafner JH, Lapotko DO. Tunable plasmonic nanobubbles for cell theranostics. Nanotechnology. 2010; 21(8):85102-85121.

10. Wagner DS, Delk NA, Lukianova-Hleb EY, Hafner JH, FarachCarson MC, Lapotko DO. The in vivo performance of plasmonic nanobubbles as cell theranostic agents in zebrafish hosting prostate cancer xenografts. Biomaterials. 2010;31(29):7567-7574.

11. Yi DK, Sun IC, Ryu JH, et al. Matrix metalloproteinase sensitive gold nanorod for simultaneous bioimaging and photothermal therapy of cancer. Bioconjug Chem. 2010;21(12):2173-2177.

12. Egeblad M, Werb Z, New functions for the matrix metalloproteinases in cancer progression. Nat Rev Cancer. 2002;2(3):161-174.

13. Bremer $\mathrm{C}$, Tung $\mathrm{CH}$, Weissleder $\mathrm{R}$. In vivo molecular target assessment of matrix metalloproteinase inhibition. Nat Med. 2001;7(6): 743-748.

14. Hu KW, Liu TM, Chung KY, et al. Efficient near-IR hyperthermia and intense nonlinear optical imaging contrast on the gold nanorod-in-shell nanostructures. J Am Chem Soc. 2009;131(40): 14186-14187.

15. Wu X, Ming T, Wang X, Wang P, Wang J, Chen J. High-photoluminescence-yield gold nanocubes: for cell imaging and photothermal therapy. ACS Nano. 2009;4(1):113-120.

16. Xia Y, Li W, Cobley CM, et al. Gold Nanocages: From Synthesis to Theranostic Applications. Acc Chem Res. 2011;44(10):914-924.

17. Moon GD, Choi SW, Cai X, et al. A new theranostic system based on gold nanocages and phase-change materials with unique features for photoacoustic imaging and controlled release. J Am Chem Soc. 2011; 133(13):4762-4765.

18. Santra S, Kaittanis C, Grimm J, Perez JM. Drug/dye-loaded, multifunctional iron oxide nanoparticles for combined targeted cancer therapy and dual optical/magnetic resonance imaging. Small. 2009; 5(16): 1862-1868.

19. Rastogi R, Gulati N, Kotnala RK, Sharma U, Jayasundar R, Koul V. Evaluation of folate conjugated pegylated thermosensitive magnetic nanocomposites for tumor imaging and therapy. Colloids Surf B Biointerfaces. 2011;82(1):160-167

20. Yallapu MM, Othman SF, Curtis ET, Gupta BK, Jaggi M, Chauhan SC. Multi-functional magnetic nanoparticles for magnetic resonance imaging and cancer therapy. Biomaterials. 2011;32(7):1890-1905.

21. Foy SP, Manthe RL, Foy ST, Dimitrijevic S, Krishnamurthy N, Labhasetwar V. Optical imaging and magnetic field targeting of magnetic nanoparticles in tumors. ACS Nano. 2010;4(9):5217-5224.

22. Lim EK, Huh YM, Yang J, Lee K, Suh JS, Haam S. pH-Triggered drug-releasing magnetic nanoparticles for cancer therapy guided by molecular imaging by MRI. Adv Mater. 2011;23(21):2436-2442.

23. Chertok B, David AE, Yang VC, Magnetically-enabled and MRmonitored selective brain tumor protein delivery in rats via magnetic nanocarriers. Biomaterials. 2011;32(26):6245-6253.

24. Singh A, Dilnawaz F, Mewar S, Sharma U, Jagannathan NR, Sahoo SK. Composite polymeric magnetic nanoparticles for co-delivery of hydrophobic and hydrophilic anticancer drugs and MRI imaging for cancer therapy. ACS Appl Mater Interfaces. 2011;3(3):842-856.

25. Das M, Mishra D, Dhak P, et al. Biofunctionalized, phosphonate-grafted, ultrasmall iron oxide nanoparticles for combined targeted cancer therapy and multimodal imaging. Small. 2009;5(24):2883-2893.

26. Mitra RN, Doshi M, Zhang X, et al. An activatable multimodal/multifunctional nanoprobe for direct imaging of intracellular drug delivery. Biomaterials. 2012;33(5):1500-1508.

27. Singh A, Dilnawaz F, Sahoo SK, Long circulating lectin conjugated paclitaxel loaded magnetic nanoparticles: a new theranostic avenue for leukemia therapy. PLoS One. 2011;6(11):26803.

28. Huang P, Li Z, Lin J, et al. Photosensitizer-conjugated magnetic nanoparticles for in vivo simultaneous magnetofluorescent imaging and targeting therapy. Biomaterials. 2011;32(13):3447-3458.

29. Park JK, Jung J, Subramaniam P, et al. Graphite-coated magnetic nanoparticles as multimodal imaging probes and cooperative therapeutic agents for tumor cells. Small. 2011;7(12):1647-1652.
30. Elsherbini AAM, Saber M, Aggag M, El-Shahawy A, Shokier HAA. Magnetic nanoparticle-induced hyperthermia treatment under magnetic resonance imaging. Magn Reson Imaging. 2011;29(2):272-280.

31. Lartigue L, Innocenti C, Kalaivani T, et al. Water-dispersible sugar-coated iron oxide nanoparticles. An evaluation of their relaxometric and magnetic hyperthermia properties. J Am Chem Soc. 2011;133(27):10459-10472.

32. Guthi JS, Yang SG, Huang G, et al. MRI-visible micellar nanomedicine for targeted drug delivery to lung cancer cells. Mol Pharm. 2009;7(1): $32-40$.

33. Cho H, Kwon GS. Polymeric micelles for neoadjuvant cancer therapy and tumor-primed optical imaging. ACS Nano. 2011;5(11):8721-8729.

34. Lu PL, Chen YC, Ou TW, et al. Multifunctional hollow nanoparticles based on graft-diblock copolymers for doxorubicin delivery. Biomaterials. 2011;32(8):2213-2221.

35. Yang X, Grailer JJ, Rowland IJ, et al. Multifunctional stable and $\mathrm{pH}$-responsive polymer vesicles formed by heterofunctional triblock copolymer for targeted anticancer drug delivery and ultrasensitive MR imaging. ACS Nano. 2010;4(11):6805-6817.

36. Xu H, Cheng L, Wang C, Ma X, Li Y, Liu Z. Polymer encapsulated upconversion nanoparticle/iron oxide nanocomposites for multimodal imaging and magnetic targeted drug delivery. Biomaterials. 2011; 32(35):9364-9373.

37. $\mathrm{Na} \mathrm{JH}, \mathrm{Koo} \mathrm{H}, \mathrm{Lee} \mathrm{S}$, et al. Real-time and non-invasive optical imaging of tumor-targeting glycol chitosan nanoparticles in various tumor models. Biomaterials. 2011;32(22):5252-5261.

38. Rungta P, Bandera YP, Roeder RD, et al. Selective imaging and killing of cancer cells with protein-activated near-infrared fluorescing nanoparticles. Macromol Biosci. 2011;11(7):927-937.

39. Santra S, Perez JM. Selective $N$-alkylation of $\beta$-alanine facilitates the synthesis of a poly(amino acid)-based theranostic nanoagent. Biomacromolecules. 2011;12(11):3917-3927.

40. Yang HM, Oh BC, Kim JH, et al. Multifunctional poly(aspartic acid) nanoparticles containing iron oxide nanocrystals and doxorubicin for simultaneous cancer diagnosis and therapy. Colloids Surf A Physicochem Eng Asp. 2011;391(1-3):208-215.

41. Kaida S, Cabral H, Kumagai M, et al. Visible drug delivery by supramolecular nanocarriers directing to single-platformed diagnosis and therapy of pancreatic tumor model. Cancer Res. 2010;70(18): 7031-7041.

42. Choi KY, Min KH, Yoon HY, et al. PEGylation of hyaluronic acid nanoparticles improves tumor targetability in vivo. Biomaterials. 2011; 32(7):1880-1889.

43. Pan J, Liu Y, Feng SS. Multifunctional nanoparticles of biodegradable copolymer blend for cancer diagnosis and treatment. Nanomedicine. 2010;5(3):347-360.

44. Kojima C, Cho SH, Higuchi E. Gold nanoparticle-loaded PEGylated dendrimers for theragnosis. Res Chem Intermed. 2012;38(6): $1279-1289$

45. He X, Wu X, Wang K, Shi B, Hai L. Methylene blue-encapsulated phosphonate-terminated silica nanoparticles for simultaneous in vivo imaging and photodynamic therapy. Biomaterials. 2009;30(29): 5601-5609.

46. Zhang R, Wu C, Tong L, Tang B, Xu QH. Multifunctional core-shell nanoparticles as highly efficient imaging and photosensitizing agents. Langmuir. 2009;25(17):10153-10158.

47. Cheng SH, Lee $\mathrm{CH}$, Chen MC, et al. Tri-functionalization of mesoporous silica nanoparticles for comprehensive cancer theranostics - the trio of imaging, targeting and therapy. J Mater Chem. 2010;20(29): 6149-6157.

48. Lu J, Liong M, Li Z, Zink JI, Tamanoi F. Biocompatibility, biodistribution, and drug-delivery efficiency of mesoporous silica nanoparticles for cancer therapy in animals. Small. 2010;6(6):1794-1805.

49. Lee JE, Lee N, Kim H, et al. Uniform mesoporous dye-doped silica nanoparticles decorated with multiple magnetite nanocrystals for simultaneous enhanced magnetic resonance imaging, fluorescence imaging, and drug delivery. J Am Chem Soc. 2009;132(2): $552-557$. 
50. Meng H, Liong M, Xia T, et al. Engineered design of mesoporous silica nanoparticles to deliver doxorubicin and P-glycoprotein siRNA to overcome drug resistance in a cancer cell line. ACS Nano. 2010; 4(8):4539-4550.

51. Robinson JT, Welsher K, Tabakman SM, et al. High performance in vivo near-IR ( $>1 \mathrm{um}$ ) imaging and photothermal cancer therapy with carbon nanotubes. Nano Res. 2010;3(11):779-793.

52. Moon HK, Lee SH, Choi HC. In vivo near-infrared mediated tumor destruction by photothermal effect of carbon nanotubes. ACS Nano. 2009;3(11):3707-3713.

53. Yang K, Zhang S, Zhang G, Sun X, Lee ST, Liu Z. Graphene in mice: ultrahigh in vivo tumor uptake and efficient photothermal therapy. Nano Lett. 2010;10(9):3318-3323.

54. Ma LL, Feldman MD, Tam JM, et al. Small multifunctional nanoclusters (nanoroses) for targeted cellular imaging and therapy. ACS Nano. 2009; 3(9):2686-2696.

55. Melancon MP, Elliott A, Ji X, et al. Theranostics with multifunctional magnetic gold nanoshells: photothermal therapy and $\mathrm{t} 2 *$ magnetic resonance imaging. Invest Radiol. 2011;46(2):132-140.

56. Ma M, Chen H, Chen Y, et al. Au capped magnetic core/mesoporous silica shell nanoparticles for combined photothermo-/chemo-therapy and multimodal imaging. Biomaterials. 2012;33(3):989-998.

57. Wang LS, Wu LC, Lu SY, et al. Biofunctionalized phospholipid-capped mesoporous silica nanoshuttles for targeted drug delivery: improved water suspensibility and decreased nonspecific protein binding. ACS Nano. 2010;4(8):4371-4379.
58. Wang C, Tao H, Cheng L, Liu Z, Near-infrared light induced in vivo photodynamic therapy of cancer based on upconversion nanoparticles. Biomaterials. 2011;32(26):6145-6154.

59. Shan J, Budijono SJ, Hu G, et al. Pegylated composite nanoparticles containing upconverting phosphors and meso-tetraphenyl porphine (TPP) for photodynamic therapy. Adv Funct Mater. 2011;21(13): 2488-2495.

60. Cheng L, Yang K, Li Y, et al. Facile preparation of multifunctional upconversion nanoprobes for multimodal imaging and dual-targeted photothermal therapy. Angew Chem Int Ed Engl. 2011;50(32): 7385-7390.

61. Medintz IL, Uyeda HT, Goldman ER, Mattoussi H. Quantum dot bioconjugates for imaging, labelling and sensing. Nat Mater. 4(6): 435-446.

62. Zhu ZJ, Yeh YC, Tang R, et al. Stability of quantum dots in live cells. Nat Chem. 2011;3(12):963-968.

63. Nurunnabi M, Cho KJ, Choi JS, Huh KM, Lee YK. Targeted near-IR QDs-loaded micelles for cancer therapy and imaging. Biomaterials. 2010;31(20):5436-5444.

64. Homan K, Shah J, Gomez S, et al. Silver nanosystems for photoacoustic imaging and image-guided therapy. J Biomed Opt. 2010;15(2): 021316.

65. Wu W, Zhou T, Berliner A, Banerjee P, Zhou S. Smart core-shell hybrid nanogels with Ag nanoparticle core for cancer cell imaging and gel shell for pH-regulated drug delivery. Chem Mater. 2010;22(6): 1966-1976.
International Journal of Nanomedicine

\section{Publish your work in this journal}

The International Journal of Nanomedicine is an international, peerreviewed journal focusing on the application of nanotechnology in diagnostics, therapeutics, and drug delivery systems throughou the biomedical field. This journal is indexed on PubMed Central, MedLine, CAS, SciSearch $₫$, Current Contents $₫ /$ Clinical Medicine,

\section{Dovepress}

Journal Citation Reports/Science Edition, EMBase, Scopus and the Elsevier Bibliographic databases. The manuscript management system is completely online and includes a very quick and fair peer-review system, which is all easy to use. Visit http://www.dovepress.com/ testimonials.php to read real quotes from published authors. 